\title{
QUEEN'S
UNIVERSITY
BELFAST
}

\section{The risk of cardiovascular disease in prostate cancer patients receiving androgen deprivation therapies}

Cardwell, C. R., O'Sullivan, J. M., Jain, S., Harbinson, M. T., Cook, M. B., Hicks, B. M., \& McMenamin, U. C. (2019). The risk of cardiovascular disease in prostate cancer patients receiving androgen deprivation therapies. Epidemiology, 31(3), 432. https://doi.org/10.1097/EDE.0000000000001132

\section{Published in:}

Epidemiology

\section{Document Version:}

Peer reviewed version

Queen's University Belfast - Research Portal:

Link to publication record in Queen's University Belfast Research Portal

\section{Publisher rights}

(c) 2019 Wolters Kluwer Health, Inc. This work is made available online in accordance with the publisher's policies. Please refer to any applicable terms of use of the publisher.

\section{General rights}

Copyright for the publications made accessible via the Queen's University Belfast Research Portal is retained by the author(s) and / or other copyright owners and it is a condition of accessing these publications that users recognise and abide by the legal requirements associated with these rights.

Take down policy

The Research Portal is Queen's institutional repository that provides access to Queen's research output. Every effort has been made to ensure that content in the Research Portal does not infringe any person's rights, or applicable UK laws. If you discover content in the Research Portal that you believe breaches copyright or violates any law, please contact openaccess@qub.ac.uk. 


\section{Type of manuscript}

Original research

\section{Title}

The risk of cardiovascular disease in prostate cancer patients receiving androgen deprivation therapies.

\section{Authors}

Chris R. Cardwell ${ }^{\mathrm{a}}$, Joe M. O’Sullivan ${ }^{\mathrm{b}, \mathrm{c}}$, Suneil Jain ${ }^{\mathrm{b}, \mathrm{c}}$, Mark T. Harbinson ${ }^{\mathrm{d}}$, Michael B.

Cook ${ }^{\mathrm{e}}$, Blánaid M. Hicks ${ }^{\mathrm{a}}$, Úna C. Mc Menaminª

\section{Authors' affiliations}

${ }^{a}$ Centre for Public Health, Queen's University Belfast, Belfast, Northern Ireland, UK.

${ }^{\mathrm{b}}$ Centre for Cancer Research and Cell Biology, Queen's University Belfast, Belfast, Northern Ireland, UK.

${ }^{\mathrm{c} R a d i o t h e r a p y}$ Department, Cancer Centre, Belfast City Hospital, Belfast, Northern Ireland, UK.

${ }^{\mathrm{d}}$ Centre for Experimental Medicine, Queen's University Belfast, Belfast, Northern Ireland, UK.

eDivision of Cancer Epidemiology and Genetics, National Cancer Institute, Bethesda, Maryland, USA.

\section{Author for correspondence}


Dr Chris R Cardwell, Institute of Clinical Sciences Block B, Queen's University Belfast, Royal Victoria Hospital, Belfast, Northern Ireland, BT12 6BA.

Telephone: +442890971649 .

Email: c.cardwell@qub.ac.uk

\section{Running title}

Androgen deprivation and cardiovascular disease.

\section{Conflicts of interest}

None declared. None of the authors has a potential conflict of interest.

\section{Source of financial support}

This work was supported by a Cancer Research UK Post-doctoral fellowship to Dr Una McMenamin [C53788/A20100] that provided access to the dataset and the Intramural Program of the National Cancer Institute, National Institutes of Health, Department of Health and Human Services, USA who fund Dr Michael B. Cook. The sponsors had no involvement with the planning, execution, or completion of the study.

\section{Data access}

Data could be accessed, at a cost, through the Information Services Division (ISD) of National Health Service (NHS) Scotland (https://www.isdscotland.org/Products-and-

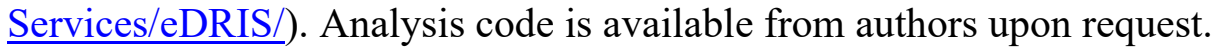

\section{Acknowledgements}

We thank the research coordinator (Lizzie Nicholson) and NHS National Services Scotland for facilitating data access and analysis. 


\section{ABSTRACT \\ Background}

Androgen deprivation therapy (ADT), which has a proven role in prostate cancer management, has been associated with various cardiovascular diseases. However few studies have investigated these associations by type of ADT, particularly for newer ADTs such as the gonadotropin-releasing hormone $(\mathrm{GnRH})$ antagonist degarelix. We investigated the risk of cardiovascular disease by type of ADT in a real world setting.

\section{Methods}

Men newly diagnosed with prostate cancer, from 2009 to 2015, were identified from the Scottish Cancer Registry. ADTs were identified from the nationwide Prescribing Information System. Cardiovascular events were based upon hospitalisation (from hospital records) or death from cardiovascular disease (from death records). Cox regression calculated hazard ratios (HRs) and 95\% confidence intervals (CIs) for cardiovascular events with time-varying ADT exposure, comparing ADT users with untreated patients, after adjusting for potential confounders including prior cardiovascular disease.

\section{Results}

The cohort contained 20,216 prostate cancer patients, followed for 73,570 person years, during which there were 3,853 cardiovascular events. ADT was associated with a $25 \%$ increase in cardiovascular events (adjusted $\mathrm{HR}=1.25$ 95\% CI 1.16, 1.35). This reflected increases in cardiovascular events associated with $\mathrm{GnRH}$ agonists (adjusted $\mathrm{HR}=1.2695 \% \mathrm{CI}$ 1.16, 1.36), degarelix (adjusted HR=1.49 95\% CI 1.16, 1.90), but not bicalutamide monotherapy (adjusted HR=1.01 95\% CI 0.82, 1.25).

\section{Conclusions}


There were increased risks of cardiovascular disease with use of GnRH agonists and degarelix, but not with bicalutamide monotherapy. This is the first study to observe increased cardiovascular risks with degarelix, but the cause of this association is unclear and merits further investigation.

\section{Keywords}

Androgen deprivation therapy, Bicalutamide, Cardiovascular Diseases, Gonadotropinreleasing hormone, Prostatic neoplasms 


\section{Introduction}

Androgen deprivation therapy (ADT) has long been used in advanced prostate cancer [1] and more recently in localised disease [2,3] with clear survival benefits alone or with radiotherapy. ADT has been linked to various side-effects including cardiovascular disease [4], for which several mechanisms have been proposed [5]. For instance, ADT increases established cardiovascular disease risk factors including obesity and dyslipidaemia [5], and ADT suppresses protective effects of androgens on atherosclerosis [5,6].

The association between ADT and cardiovascular disease remains controversial. Since 2010 the US Food and Drug Administration has required manufacturers of GnRH (gonadotropin-releasing hormone) agonists to include safety information warning of increased risks of cardiovascular disease [7]. This is consistent with a 2015 meta-analysis of observational studies (including 6 studies of GnRH agonists and 3 of antiandrogens) which observed increases in cardiovascular disease [8] but not a 2011 meta-analysis of trials that did not show increases in fatal cardiovascular disease [9]. The different findings could reflect confounding in observational studies or the inclusion of heathier trial populations [9].

Recently authors have suggested that the different mechanisms of specific ADTs could alter cardiovascular disease risk [5]. A post-hoc analysis of a systematic review of trials suggested that the GnRH antagonist degarelix had a weaker adverse effect on cardiovascular disease, compared with GnRH agonists [10]. However only one observational study [7] which had limited statistical power due to only 12 ischaemic events in degarelix users has investigated this association.

Therefore, we investigated the risk of cardiovascular disease by type of ADT in a large population-based prostate cancer cohort. 


\section{Methods}

Data sources

The study utilised the following linked databases [11]: the Scottish Cancer Registry; the Scottish National Prescribing Information System (PIS), which captures all community dispensed medications in Scotland; the General / Acute Inpatient and Day Case dataset (SMR01), which captures hospital diagnoses and operations; the Outpatient Attendance dataset (SMR00), which captures diagnoses and procedures from outpatient clinics; and, the National Records of Scotland Death Records which captures date and cause of death. These databases covered Scotland from January 1999 to July 2017, apart from the PIS which was available from January 2009 to July 2017. Linkages between data sources were conducted using the Community Health Index number (unique to each Scottish resident) [11]. The study was approved by the Privacy Advisory Committee of the National Health Service (NHS) National Services Scotland (number:1617-0374).

Study design

A cohort of men newly diagnosed with prostate cancer (ICD10, International Classification of Diseases $10^{\text {th }}$ revision, code C61) between January 2009 and December 2015, was identified from the Scottish Cancer Registry. Men were excluded if they: had a previous cancer diagnosis (apart from non-melanoma skin cancer or in-situ tumours); had inconsistent dates (specifically a record of ADT or radical prostatectomy, RP, more than 6 months prior to prostate cancer diagnosis).

The primary outcome was a cardiovascular event consisting of either death from cardiovascular disease or hospitalisation for cardiovascular disease. Death from cardiovascular disease was identified by ICD10 codes I20 to I99 and G45, as the underlying cause of death, within National Records of Scotland Death Records up to July 2017. 
Hospitalisation for cardiovascular disease was identified, using the same ICD10 codes, from the primary condition for hospitalisation within SMR01 to July 2017. Analysis were also conducted by cardiovascular disease type using previous definitions for angina (I20, I24, I25 [12]), acute myocardial infarction (I21, I22 [12]), stroke (I60-64, G45 [13]), venous thromboembolism (I26, I80, I82.1-2.9 [14]), heart failure (I50, I11.0 [12]), arrhythmia (I4449[13]) and other cardiovascular disease (all remaining I codes not previously categorised).

\section{Exposure}

ADT consisted of GnRH agonists (including goserelin, leuprorelin, triptorelin, and histrelin), oral antiandrogens (including bicalutamide, enzalutamide, flutamide and cyproterone acetate), the GnRH antagonist degarelix, estrogens and orchidectomy. Medical ADT was identified from dispensed medications from the PIS. The pack size and strength was used to calculate the number of days use based upon the daily defined doses (DDDs) as given by the World Health Organisation [15]. The Scottish Cancer Registry provided data on initial curative radiotherapy, RT. Orchidectomy (ICD10 codes N051, N052, N061 and N063 [16]) and RP (code M61 [16]) were taken from SMR01.

\section{Confounders}

The Scottish Cancer Registry provided Gleason score, and stage (based upon pathological stage, where recorded, or clinical stage). Cardiovascular diseases named previously and other comorbidities from the Charlson comorbidity index (specifically dementia, pulmonary disease, peptic ulcer, liver disease, diabetes, diabetes complications, paraplegia, connective tissue disorder, renal disease and severe liver disease, using previous ICD10 codes [17]) were identified before prostate cancer diagnosis from hospital admissions data (SMR00 and SMR01). The following medications were determined from PIS records in 
the year prior to diagnosis: non-steroidal anti-inflammatory drugs (NSAIDs), aspirin, betablockers, angiotensin-converting enzyme inhibitors (ACEIs), angiotensin II receptor blockers (ARBs), diuretics, statins, warfarin, digoxin, clopidogrel, dipyridamole, nitrate, insulin, sulfonylureas, metformin and other diabetic medications. Deprivation level was determined from postcode of residence using the 2009 Scottish Index of Multiple Deprivation [18].

Statistical analysis

In the primary analysis, prostate cancer patients were followed from prostate cancer diagnosis to the earliest of the date of first cardiovascular event, date of death, date of leaving Scotland or July 2017. Treatment was coded as a single time-varying and hierarchical variable to reflect treatment pathways [19,20]. Specifically, participants were initially in the untreated group and entered the ADT group on the date of their first dispensed ADT (regardless of future or past RP or RT) and remained in the ADT group for the rest of followup. Participants entered the RP group on the date of RP (where RP occurred before first ADT) and remained in the RP group for the rest of follow-up or until the date of first ADT. Participants entered the RT group on the date of RT (where RT occurred before ADT) and remained in the RT group for the rest of follow-up or until the date of first ADT or RP (see Figure 1A).

Cox regression models were used to calculate hazard ratios (HRs) and 95\% confidence intervals $(95 \% \mathrm{CI})$ for cardiovascular events comparing the ADT, RT and RP exposure to untreated patients (receiving active surveillance or watchful waiting). The main model contained year of diagnosis, age, deprivation, comorbidities (stated above, at any time before diagnosis) and other medications (stated above, in the year before diagnosis using separate variables for each). Separate analyses, restricted to patients diagnosed 2012 to 2015, when stage was routinely recorded, were conducted additionally adjusted for stage (including 
stage X[unstaged/stage unknown] as a category) and Gleason score (restricted to those with available data). A separate analysis was also conducted using a complete case analysis to adjust for stage and Gleason score, treating stage $\mathrm{X}$ as missing. In dose response analysis using a time-varying covariate, patients were deemed non-users prior to their first dispensed ADT, a short-term user from this time to 365 days of use (based upon DDDs) and a longterm user from then on (see Figure 1B). Analyses were repeated with ADT type coded as a single time-varying and hierarchical variable into the following hierarchical categories (to reflect treatment pathways[19,20]): GnRH agonists (with or without other ADT), degarelix (with or without antiandrogens, oestrogen or orchidectomy), bicalutamide $150 \mathrm{mg}$ (analysed separately as predominantly used as monotherapy; with or without other antiandrogens, oestrogens or orchidectomy) and other antiandrogens (with or without oestrogens or orchidectomy).

Additional analyses were conducted. First, analyses were repeated using a primary outcome of death from cardiovascular disease only and, separately, repeated for cardiovascular disease incidence. Second, analyses were repeated stratifying by stage, age and cardiovascular disease before diagnosis. Third, analyses were conducted in which ADT users who subsequently received RT were allocated to a separate "ADT then RT" exposure category on the date of RT receipt. Fourth, analyses were conducted on an "intention-totreat" exposure model with treatment groups based solely upon initial treatment (except for individuals simultaneously starting GnRH and antiandrogens who were allocated to GnRH exposure, as such antiandrogens were likely used short-term for tumour flare). Fifth, avoiding hierarchical exposure assignment, a separate analysis was conducted comparing each ADT individually ( $\mathrm{GnRH}$, degarelix, bicalutamide and other antiandrogens) with no treatment, regardless of use of another ADT. Sixth, analyses were repeated with death based upon a 
cardiovascular disease code as any cause of death, and incidence based upon a cardiovascular disease code anywhere in SMR00 and SMR01.

Current use of ADT was investigated using a start/stop time-varying exposure in which patients became a user upon the date of each dispensed ADT and remained a user for the duration of the prescription, based upon the DDD, plus a residual effect period. The duration of the residual effect was taken from a previous study [21] and was 3 months for GnRH agonists and antagonists and 1 month for antiandrogens and estrogens (see Figure 1C). The exposure period between the end of one prescription and start of the next was allocated to past use. All analyses were conducted using STATA 14 (StataCorp, College Station, TX, USA). 


\section{Results}

Overall 22,366 patients were identified with prostate cancer between 2009 and 2015. Of these 1,839 had prior cancer, 169 had date inconsistencies, 142 had events on the date of prostate cancer diagnosis. The final cohort contained 20,216 prostate cancer patients, 73,570 person years, and 3,853 cardiovascular events (52 per 1,000 person years).

Patient Characteristics

ADT users, compared with untreated patients, were older, less deprived, and had higher stage and Gleason score (see Table 1). ADT users had similar rates of medication use and cardiovascular disease before diagnosis, compared with untreated patients.

ADT and cardiovascular events

Table 2 shows, compared with untreated patients, the rates of cardiovascular events were similar in the radical prostatectomy and radiotherapy groups but higher in ADT users (adjusted HR=1.25 95\% CI 1.16, 1.35). More marked associations (adjusted HR=1.36 95\% CI 1.24, 1.48) were seen after receiving more than a year supply, 365 DDDs, of ADT. An increase in cardiovascular events was seen for $\mathrm{GnRH}$ agonists (adjusted $\mathrm{HR}=1.26$ 95\% CI 1.16, 1.36), degarelix (adjusted HR=1.49 95\% CI 1.16, 1.90), non-bicalutamide antiandrogens (adjusted HR=1.30 95\% CI 1.07, 1.57) but not bicalutamide (adjusted $\mathrm{HR}=1.0195 \% \mathrm{CI} 0.82,1.25)$. The difference between unadjusted and adjusted results partly reflected adjustment for age (see eTable 1). These associations were similar after further adjustment for stage and Gleason score and when based solely upon incidence of cardiovascular disease, but were slightly attenuated when based upon death from cardiovascular disease (eTable 2 and 3 ) 
Table 3 shows analysis by specific cardiovascular events. Overall, ADT users compared with untreated patients had higher risks of myocardial infarction (adjusted $\mathrm{HR}=1.23$ 95\% CI 1.03, 1.46), arrhythmia (adjusted HR=1.21 95\% CI 1.01, 1.45), venous thromboembolism (adjusted HR=2.28 95\% CI 1.76, 2.97) and unclassified other cardiovascular events (adjusted HR=1.28 95\% CI 1.11, 1.47). These associations largely reflected GnRH agonists, but numbers were limited to investigate ADT types. After further adjustment for stage and Gleason score (shown in eTable 4) associations were similar, except for venous thromboembolism (adjusted $\mathrm{HR}=1.26$ 95\% CI 0.76, 2.09).

Secondary $\backslash$ sensitivity analyses

The association between ADT and cardiovascular events appeared similar in patients with and without prior cardiovascular disease (adjusted HR=1.22 95\% CI 1.09, 1.36 and 1.34 95\% CI 1.21, 1.49, see Table 4), by stage (see eTable 5) and by age (see eTable 6). Treating stage $\mathrm{X}$ as missing, and analysing the data as complete case had little impact on the results (see eTable 7). The associations were little altered after analyses assigning RT to a separate category, analyses based upon "intention-to-treat", or analyses comparing each ADT individually with no treatment, regardless of use of another ADT (see eTables 8 and 9). Associations were generally attenuated when analyses were repeated with death based upon cardiovascular disease as any cause of death, and incidence based upon cardiovascular disease codes anywhere in hospital admissions data (see eTable 10).

Finally, current use of ADT (adjusted HR=1.33 95\% CI 1.23, 1.44) was associated with cardiovascular events whilst past use (adjusted HR=1.06 95\% CI 0.95, 1.18) was not, see Table 5. Current ADT use had generally similar associations with specific cardiovascular diseases as any ADT use (see eTable 11). 


\section{Discussion}

We observed approximately a $25 \%$ increase in the risk of cardiovascular events in ADT users compared with untreated patients that mainly reflected increases for GnRH agonists and the GnRH antagonist degarelix. These associations followed a dose response and were apparent for current, but not past, ADT use. There was generally no association between bicalutamide use and cardiovascular events. ADT was associated with increased risks of venous thromboembolism, myocardial infarction and arrhythmia, but these associations were not robust across all analyses.

Our main ADT findings are largely consistent with a 2015 meta-analysis of observational studies that observed evidence of around $40 \%$ increases in cardiovascular disease risk with GnRH agonist based upon 6 studies [8]. Furthermore, since then additional studies have been conducted comparing ADT users to non-users which have shown increased risks of cardiovascular disease by $37 \%$ [22], increased risk of heart failure by $27 \%$, arrhythmia by $17 \%[12]$ and venous thromboembolism by $84 \%$ [14].

The only previous observational study investigating cardiovascular disease risk with degarelix observed no difference in the risk of myocardial infarction or stroke in degarelix users compared with GnRH antagonists [7]. Similarly, we observed an increased risk of cardiovascular events with degarelix use similar to that seen for GnRH agonist use, compared with untreated patients. Furthermore, this pattern was similar in patients with pre-existing cardiovascular disease although our power was reduced in this group. Consequently, our findings do not appear to support a meta-analysis of trials suggesting that degarelix had a lower cardiovascular disease risk, compared with GnRH agonists in patients with pre-existing cardiovascular disease [10]. This difference in findings could reflect confounding within our study. For instance, degarelix users have more advanced prostate cancer which could increase cardiovascular disease risk and although we adjusted for stage and grade, these 
variables were not complete. Alternatively, the difference could reflect Type 1 error or the inclusion of a real world population in our study with fewer comorbidity restrictions. Further research on the association between degarelix and cardiovascular events is merited and a trial is ongoing but is not due to report until 2021 [23].

We observed, in general, lower cardiovascular disease risk with bicalutamide monotherapy, which could reflect higher testosterone levels [24]. This should provide some reassurance to clinicians using bicalutamide in locally advanced non-metastatic prostate cancer [25] and complements studies which have shown a reduced impact of bicalutamide on bone health [24] and comorbidities more generally [26].

Strengths of our study include use of a large cohort of prostate cancer patients with high completeness[27] and detailed information on use and timing of ADT and access to a wide range of confounders including prior cardiovascular disease, cancer stage and Gleason score. Cardiovascular events were based upon National death and hospital records, which have high accuracy for various conditions including cardiovascular diseases [28], and of reassurance, results were similar when incidence or mortality data were analysed separately.

There is the possibility of residual confounding by incompletely recorded (stage, Gleason score) or unavailable variables (such as lifestyle cardiovascular disease risk factors including obesity, smoking or alcohol intake). ADT use was determined from dispensing records and medication adherence cannot be confirmed. However, the limited evidence that exists, suggests high adherence to oral ADT medications [29]. Finally, patients receiving ADT may have increased exposure to health care professionals increasing the likelihood of cardiovascular disease being identified.

In conclusion, GnRH agonists and degarelix were associated with increased cardiovascular disease risk. This is the first study to observe increased risks of 
cardiovascular disease with degarelix. The cause of this association is unclear and merits further investigation. 
Table 1. Characteristics of prostate cancer cohort by treatment group.

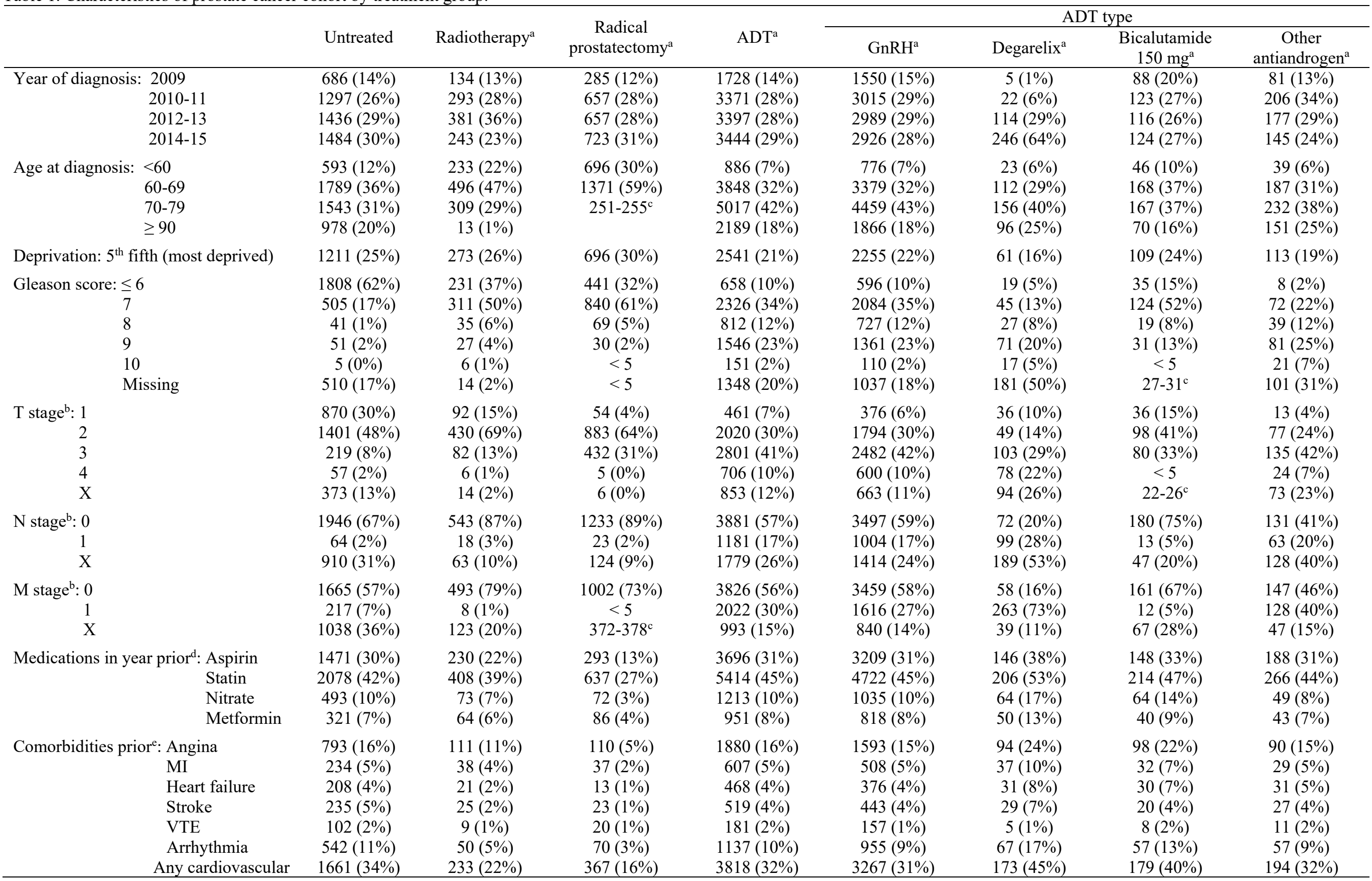


MI. myocardial infarction; VTE, venous thromboembolism.

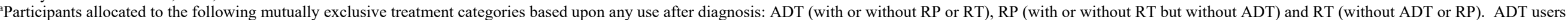

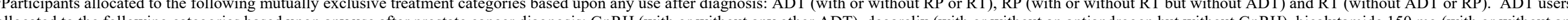
allocated to he following catgories based upon any use afer prostate cancer diagnosis. GnRH (with or without any or (WDT),

${ }^{d}$ Medications at any time prior to prostate cancer diagnosis

${ }^{\circ}$ Comorbidities at any time prior to prostate cancer diagnosis. 
Table 2. Association between androgen deprivation therapy and cardiovascular events.

\begin{tabular}{|c|c|c|c|c|c|c|c|c|c|c|c|c|c|}
\hline & & Case & $\begin{array}{c}\text { Person } \\
\text { years }\end{array}$ & $\begin{array}{c}\text { Unadjusted HR } \\
(95 \% \mathrm{CI})\end{array}$ & $\mathrm{P}$ & $\begin{array}{c}\text { Adjusted }^{\mathrm{a}} \mathrm{HR} \\
(95 \% \mathrm{CI})\end{array}$ & $\mathrm{P}$ & Case & $\begin{array}{c}\text { Person } \\
\text { years }\end{array}$ & $\begin{array}{c}\text { Unadjusted HR } \\
(95 \% \mathrm{CI})\end{array}$ & $\mathrm{P}$ & $\begin{array}{c}\text { Adjusted }^{\mathrm{b}} \mathrm{HR} \\
(95 \% \mathrm{CI})\end{array}$ & $\mathrm{P}$ \\
\hline & & \multicolumn{6}{|c|}{ Diagnosed 2009 to 2015} & \multicolumn{6}{|c|}{ Diagnosed 2012 to 2015 (additionally adjusted for stage and Gleason) } \\
\hline \multicolumn{2}{|l|}{ Untreated } & 1105 & 22590 & 1.00 (ref. cat.) & & 1.00 (ref. cat.) & & 511 & 10837 & 1.00 (ref. cat.) & & 1.00 (ref. cat.) & \\
\hline \multicolumn{2}{|c|}{ Radical prostatectomy } & 253 & 9350 & $0.61(0.53,0.70)$ & $<0.001$ & $0.94(0.81,1.09)$ & 0.404 & 104 & 3836 & $0.63(0.51,0.78)$ & $<0.001$ & $0.98(0.77,1.24)$ & 0.847 \\
\hline \multicolumn{2}{|l|}{ Radiotherapy } & 135 & 4450 & $0.68(0.57,0.82)$ & $<0.001$ & $0.85(0.71,1.02)$ & 0.076 & 53 & 1929 & $0.65(0.49,0.86)$ & 0.003 & $0.82(0.61,1.11)$ & 0.206 \\
\hline \multirow[t]{2}{*}{ ADT: } & Any use & 2360 & 37180 & $1.38(1.28,1.49)$ & $<0.001$ & $1.25(1.16,1.35)$ & $<0.001$ & 1069 & 16562 & $1.46(1.31,1.64)$ & $<0.001$ & $1.32(1.12,1.54)$ & 0.001 \\
\hline & 365+ DDDs & 1381 & 20685 & $1.56(1.43,1.70)$ & $<0.001$ & $1.36(1.24,1.48)$ & $<0.001$ & 576 & 8303 & $1.73(1.51,1.98)$ & $<0.001$ & $1.59(1.31,1.93)$ & $<0.001$ \\
\hline \multirow[t]{3}{*}{ GnRH: } & Any use & 2075 & 33023 & $1.37(1.27,1.48)$ & $<0.001$ & $1.26(1.16,1.36)$ & $<0.001$ & 920 & 14575 & $1.43(1.28,1.60)$ & $<0.001$ & $1.33(1.13,1.56)$ & 0.001 \\
\hline & 0-365 DDDs & 990 & 16606 & $1.24(1.14,1.36)$ & $<0.001$ & $1.18(1.08,1.29)$ & $<0.001$ & 487 & 8101 & $1.31(1.15,1.49)$ & $<0.001$ & $1.26(1.06,1.49)$ & 0.007 \\
\hline & 365+ DDDs & 1085 & 16417 & $1.55(1.41,1.69)$ & $<0.001$ & $1.36(1.24,1.49)$ & $<0.001$ & 433 & 6473 & $1.65(1.43,1.90)$ & $<0.001$ & $1.55(1.26,1.89)$ & $<0.001$ \\
\hline \multirow[t]{3}{*}{ Degarelix: } & Any use & 71 & 726 & $2.01(1.58,2.56)$ & $<0.001$ & $1.49(1.16,1.90)$ & 0.001 & 64 & 657 & $2.12(1.63,2.76)$ & $<0.001$ & $1.47(0.99,2.18)$ & 0.054 \\
\hline & 0-365 DDDs & 41 & 393 & $1.98(1.45,2.71)$ & $<0.001$ & $1.45(1.06,2.00)$ & 0.02 & 37 & 361 & $2.05(1.47,2.88)$ & $<0.001$ & $1.33(0.79,2.24)$ & 0.28 \\
\hline & 365+ DDDs & 30 & 333 & $2.01(1.39,2.89)$ & $<0.001$ & $1.52(1.05,2.19)$ & 0.026 & 27 & 296 & $2.20(1.49,3.26)$ & $<0.001$ & $1.81(1.05,3.12)$ & 0.032 \\
\hline \multirow[t]{3}{*}{ Bicalutamide: } & Any use & 95 & 1711 & $1.22(0.98,1.50)$ & 0.07 & $1.01(0.82,1.25)$ & 0.913 & 37 & 620 & $1.35(0.97,1.89)$ & 0.079 & $1.13(0.77,1.65)$ & 0.524 \\
\hline & 0-365 DDDs & 33 & 539 & $1.24(0.88,1.76)$ & 0.219 & $1.08(0.76,1.53)$ & 0.67 & 15 & 250 & $1.28(0.76,2.14)$ & 0.35 & $0.97(0.52,1.83)$ & 0.929 \\
\hline & $365+$ DDDs & 62 & 1172 & $1.22(0.94,1.58)$ & 0.13 & $0.99(0.76,1.28)$ & 0.939 & 22 & 370 & $1.44(0.93,2.21)$ & 0.098 & $1.29(0.82,2.03)$ & 0.279 \\
\hline \multicolumn{14}{|c|}{ Other antiandrogen: } \\
\hline & Any use & 117 & 1659 & $1.53(1.26,1.85)$ & $<0.001$ & $1.30(1.07,1.57)$ & 0.008 & 47 & 697 & $1.52(1.13,2.06)$ & 0.006 & $1.27(0.85,1.91)$ & 0.238 \\
\hline & 0-365 DDDs & 103 & 1520 & $1.47(1.20,1.80)$ & $<0.001$ & $1.28(1.04,1.56)$ & 0.019 & 42 & 667 & $1.43(1.04,1.97)$ & 0.026 & $1.25(0.82,1.91)$ & 0.303 \\
\hline & $365+$ DDDs & 14 & 139 & $2.43(1.43,4.12)$ & 0.001 & $1.57(0.92,2.67)$ & 0.099 & 5 & 30 & $4.21(1.74,10.18)$ & 0.001 & $2.98(0.94,9.48)$ & 0.064 \\
\hline
\end{tabular}

${ }^{a}$ Model contains year of diagnosis, age, deprivation, comorbidities in year before prostate cancer diagnosis (myocardial infarction, angina, cerebral vascular accident, heart failure, venous thromboembolism,

arrhythmia, other cardiovascular disease, dementia, pulmonary disease, connective tissue disorder, peptic ulcer, liver disease, diabetes, diabetes complications, paraplegia, severe liver disease) and other medication use in the year before prostate cancer diagnosis (including separately aspirin, beta-blockers, ACEIs, ARBs, diuretics, statins, warfarin, digoxin, clopidogrel, dipyridamole, nitrate, insulin, sulfonylureas, metformin and other diabetic medications). ${ }^{\mathrm{b}}$ Adjusted models contain all terms in ${ }^{\mathrm{a}}$ as well as $\mathrm{t}$ stage, $\mathrm{n}$ stage, $\mathrm{m}$ stage and Gleason score. 
Table 3. Association between androgen deprivation therapy and type of cardiovascular events in patients diagnosed 2009 to 2015.

\begin{tabular}{|c|c|c|c|c|c|c|}
\hline & Case & $\begin{array}{c}\text { Person } \\
\text { years }\end{array}$ & $\begin{array}{c}\text { Unadjusted HR } \\
(95 \% \mathrm{CI})\end{array}$ & $\mathrm{P}$ & $\begin{array}{c}\text { Adjusted }^{\mathrm{a}} \mathrm{HR} \\
(95 \% \mathrm{CI})\end{array}$ & $\mathrm{P}$ \\
\hline \multicolumn{7}{|c|}{ Angina } \\
\hline Untreated & 260 & 24089 & 1.00 (ref. cat.) & & 1.00 (ref. cat.) & \\
\hline ADT & 510 & 40923 & $1.13(0.97,1.32)$ & 0.122 & $1.02(0.88,1.20)$ & 0.769 \\
\hline $\mathrm{GnRH}$ & 440 & 36370 & $1.09(0.93,1.28)$ & 0.261 & $1.01(0.86,1.19)$ & 0.885 \\
\hline Degaralex & 16 & 818 & $1.71(1.03,2.84)$ & 0.038 & $1.07(0.64,1.79)$ & 0.801 \\
\hline Bicalutamide & 30 & 1853 & $1.48(1.01,2.17)$ & 0.042 & $1.13(0.77,1.65)$ & 0.537 \\
\hline Other antiandrogen & 24 & 1816 & $1.20(0.79,1.83)$ & 0.393 & $1.14(0.75,1.73)$ & 0.546 \\
\hline \multicolumn{7}{|c|}{ Myocardial infarction } \\
\hline Untreated & 201 & 24308 & 1.00 (ref. cat.) & & 1.00 (ref. cat.) & \\
\hline ADT & 440 & 41352 & $1.38(1.16,1.64)$ & $<0.001$ & $1.23(1.03,1.46)$ & 0.023 \\
\hline $\mathrm{GnRH}$ & 386 & 36759 & $1.36(1.14,1.63)$ & 0.001 & $1.23(1.03,1.47)$ & 0.025 \\
\hline Degaralex & 12 & 819 & $1.83(1.02,3.28)$ & 0.043 & $1.26(0.70,2.29)$ & 0.442 \\
\hline Bicalutamide & 26 & 1869 & $1.79(1.19,2.71)$ & 0.005 & $1.46(0.97,2.21)$ & 0.073 \\
\hline Other antiandrogen & 16 & 1841 & $1.12(0.67,1.88)$ & 0.652 & $0.95(0.57,1.59)$ & 0.855 \\
\hline \multicolumn{7}{|c|}{ Arrhythmia } \\
\hline Untreated & 184 & 24221 & 1.00 (ref. cat.) & & 1.00 (ref. cat.) & \\
\hline ADT & 414 & 41064 & $1.33(1.11,1.59)$ & 0.002 & $1.21(1.01,1.45)$ & 0.038 \\
\hline $\mathrm{GnRH}$ & 357 & 36501 & $1.29(1.07,1.54)$ & 0.007 & $1.19(0.99,1.43)$ & 0.069 \\
\hline Degaralex & 14 & 809 & $2.27(1.32,3.92)$ & 0.003 & $1.55(0.89,2.72)$ & 0.121 \\
\hline Bicalutamide & 17 & 1879 & $1.19(0.72,1.96)$ & 0.5 & $1.01(0.61,1.67)$ & 0.957 \\
\hline Other antiandrogen & 25 & 1811 & $1.83(1.20,2.78)$ & 0.005 & $1.64(1.07,2.49)$ & 0.022 \\
\hline \multicolumn{7}{|c|}{ Heart failure } \\
\hline Untreated & 104 & 24454 & 1.00 (ref. cat.) & & 1.00 (ref. cat.) & \\
\hline ADT & 229 & 41639 & $1.41(1.11,1.80)$ & 0.005 & $1.21(0.95,1.55)$ & 0.12 \\
\hline $\mathrm{GnRH}$ & 197 & 37018 & $1.37(1.07,1.76)$ & 0.014 & $1.21(0.94,1.55)$ & 0.142 \\
\hline Degaralex & 8 & 821 & $2.23(1.08,4.60)$ & 0.03 & $1.22(0.58,2.58)$ & 0.597 \\
\hline Bicalutamide & 16 & 1881 & $2.20(1.29,3.73)$ & 0.004 & $1.87(1.10,3.20)$ & 0.021 \\
\hline Other antiandrogen & 8 & 1854 & $1.10(0.54,2.27)$ & 0.787 & $0.80(0.39,1.65)$ & 0.547 \\
\hline \multicolumn{7}{|c|}{ Stroke } \\
\hline Untreated & 226 & 24198 & 1.00 (ref. cat.) & & 1.00 (ref. cat.) & \\
\hline $\mathrm{ADT}$ & 437 & 41206 & $1.15(0.98,1.36)$ & 0.093 & $1.04(0.88,1.22)$ & 0.682 \\
\hline $\mathrm{GnRH}$ & 394 & 36621 & $1.17(0.99,1.39)$ & 0.068 & $1.06(0.90,1.26)$ & 0.489 \\
\hline Degaralex & 6 & 818 & $0.80(0.35,1.80)$ & 0.586 & $0.58(0.25,1.31)$ & 0.187 \\
\hline Bicalutamide & 12 & 1883 & $0.69(0.39,1.24)$ & 0.214 & $0.62(0.35,1.12)$ & 0.112 \\
\hline Other antiandrogen & 24 & 1822 & $1.44(0.94,2.19)$ & 0.094 & $1.18(0.77,1.81)$ & 0.436 \\
\hline \multicolumn{7}{|c|}{ Venous thromboembolism } \\
\hline Untreated & 89 & 24459 & 1.00 (ref. cat.) & & 1.00 (ref. cat.) & \\
\hline ADT & 273 & 41460 & $2.34(1.80,3.03)$ & $<0.001$ & $2.28(1.76,2.97)$ & $<0.001$ \\
\hline $\mathrm{GnRH}$ & 243 & 36837 & $2.35(1.80,3.05)$ & $<0.001$ & $2.31(1.77,3.01)$ & $<0.001$ \\
\hline Degaralex & 10 & 814 & $3.85(1.99,7.45)$ & $<0.001$ & $3.74(1.91,7.32)$ & $<0.001$ \\
\hline Bicalutamide & 5 & 1902 & $0.92(0.37,2.27)$ & 0.852 & $0.86(0.35,2.13)$ & 0.745 \\
\hline Other antiandrogen & 15 & 1842 & $2.78(1.60,4.83)$ & $<0.001$ & $2.64(1.51,4.61)$ & 0.001 \\
\hline \multicolumn{7}{|c|}{ Other CVD events } \\
\hline Untreated & 320 & 23967 & 1.00 (ref. cat.) & & 1.00 (ref. cat.) & \\
\hline ADT & 726 & 40390 & $1.38(1.20,1.58)$ & $<0.001$ & $1.28(1.11,1.47)$ & $<0.001$ \\
\hline GnRH & 657 & 35853 & $1.41(1.22,1.62)$ & $<0.001$ & $1.32(1.14,1.52)$ & $<0.001$ \\
\hline Degaralex & 22 & 804 & $2.07(1.34,3.20)$ & 0.001 & $1.73(1.11,2.68)$ & 0.015 \\
\hline Bicalutamide & 19 & 1843 & $0.79(0.50,1.26)$ & 0.332 & $0.70(0.44,1.11)$ & 0.133 \\
\hline Other antiandrogen & 28 & 1826 & $1.17(0.80,1.73)$ & 0.418 & $1.02(0.69,1.51)$ & 0.912 \\
\hline
\end{tabular}


dementia, pulmonary disease, connective tissue disorder, peptic ulcer, liver disease, diabetes, diabetes complications, paraplegia, severe liver disease) and other medication use in the year before prostate cancer diagnosis (including separately aspirin, betablockers, ACEIs, ARBs, diuretics, statins, warfarin, digoxin, clopidogrel, dipyridamole, nitrate, insulin, sulfonylureas, metformin and other diabetic medications). 
Table 4. Association between androgen deprivation therapy and cardiovascular events stratified by previous cardiovascular disease.

\begin{tabular}{|c|c|c|c|c|c|c|c|c|c|c|c|c|}
\hline & Case & $\begin{array}{c}\text { Person } \\
\text { years }\end{array}$ & $\begin{array}{c}\text { Unadjusted HR } \\
(95 \% \mathrm{CI})\end{array}$ & $\mathrm{P}$ & $\begin{array}{c}\text { Adjusted }{ }^{a} \mathrm{HR} \\
(95 \% \mathrm{CI})\end{array}$ & $\mathrm{P}$ & Case & $\begin{array}{c}\text { Person } \\
\text { years }\end{array}$ & $\begin{array}{c}\text { Unadjusted HR } \\
(95 \% \mathrm{CI})\end{array}$ & $\mathrm{P}$ & $\begin{array}{c}\text { Adjusted }{ }^{b} \mathrm{HR} \\
(95 \% \mathrm{CI})\end{array}$ & $\mathrm{P}$ \\
\hline & \multicolumn{6}{|c|}{ Diagnosed 2009 to 2015} & \multicolumn{6}{|c|}{ Diagnosed 2012 to 2015 (additionally adjusted for stage and Gleason) } \\
\hline & & & & & CVD prior & & & & & & & \\
\hline Untreated & 555 & 5950 & 1.00 (ref. cat.) & & 1.00 (ref. cat.) & & 268 & 3001 & 1.00 (ref. cat.) & & 1.00 (ref. cat.) & \\
\hline ADT & 1074 & 10281 & $1.21(1.09,1.35)$ & 0.001 & $1.14(1.02,1.27)$ & 0.017 & 537 & 4890 & $1.31(1.12,1.53)$ & 0.001 & $1.35(1.07,1.69)$ & 0.01 \\
\hline GnRH & 931 & 8934 & $1.21(1.08,1.35)$ & 0.001 & $1.16(1.04,1.30)$ & 0.009 & 451 & 4183 & $1.29(1.10,1.52)$ & 0.002 & $1.34(1.06,1.69)$ & 0.013 \\
\hline Degaralex & 40 & 287 & $1.51(1.09,2.08)$ & 0.012 & $1.22(0.88,1.69)$ & 0.239 & 35 & 264 & $1.51(1.06,2.16)$ & 0.023 & $1.46(0.88,2.41)$ & 0.14 \\
\hline Bicalutamide & 54 & 573 & $1.10(0.83,1.46)$ & 0.494 & $0.97(0.73,1.29)$ & 0.844 & 29 & 243 & $1.45(0.98,2.13)$ & 0.062 & $1.38(0.88,2.15)$ & 0.159 \\
\hline \multicolumn{13}{|c|}{ No CVD prior } \\
\hline Untreated & 550 & 16639 & 1.00 (ref. cat.) & & 1.00 (ref. cat.) & & 243 & 7836 & 1.00 (ref. cat.) & & 1.00 (ref. cat.) & \\
\hline ADT & 1286 & 26899 & $1.50(1.36,1.67)$ & $<0.001$ & $1.34(1.21,1.49)$ & $<0.001$ & 532 & 11672 & $1.55(1.32,1.82)$ & $<0.001$ & $1.27(1.02,1.59)$ & 0.033 \\
\hline GnRH & 1144 & 24088 & $1.49(1.34,1.66)$ & $<0.001$ & $1.33(1.20,1.48)$ & $<0.001$ & 469 & 10392 & $1.53(1.30,1.80)$ & $<0.001$ & $1.31(1.04,1.63)$ & 0.02 \\
\hline Degaralex & 31 & 440 & $2.18(1.52,3.14)$ & $<0.001$ & $1.82(1.26,2.63)$ & 0.001 & 29 & 393 & $2.47(1.68,3.65)$ & $<0.001$ & $1.51(0.79,2.87)$ & 0.213 \\
\hline Bicalutamide & 41 & 1138 & $1.13(0.82,1.56)$ & 0.446 & $1.01(0.74,1.39)$ & 0.942 & 8 & 378 & $0.72(0.35,1.45)$ & 0.352 & $0.67(0.31,1.44)$ & 0.309 \\
\hline Other antiandrogen & 68 & 1191 & $1.79(1.39,2.30)$ & $<0.001$ & $1.61(1.25,2.07)$ & $<0.001$ & 25 & 506 & $1.67(1.10,2.53)$ & 0.016 & $1.28(0.75,2.21)$ & 0.365 \\
\hline
\end{tabular}

${ }^{a}$ Model contains for year of diagnosis, age, deprivation, comorbidities in the year before prostate cancer diagnosis (myocardial infarction, angina, cerebral vascular accident, heart failure, venous thromboembolism,

arrhythmia, other cardiovascular disease, dementia, pulmonary disease, connective tissue disorder, peptic ulcer, liver disease, diabetes, diabetes complications, paraplegia, severe liver disease) and other medication use

in the year before prostate cancer diagnosis (including separately aspirin, beta-blockers, ACEIs, ARBs, diuretics, statins, warfarin, digoxin, clopidogrel, dipyridamole, nitrate, insulin, sulfonylureas, metformin and

othaer diabetic medications). ${ }^{b}$ Adjusted models contain all terms in ${ }^{a}$ as well as t stage, $\mathrm{n}$ stage, $\mathrm{m}$ stage and Gleason score. 
Table 5. Association between current use of androgen deprivation therapy and cardiovascular events.

\begin{tabular}{|c|c|c|c|c|c|c|c|c|c|c|c|c|c|}
\hline & & Case & $\begin{array}{l}\text { Person } \\
\text { years }\end{array}$ & $\begin{array}{c}\text { Unadjusted HR } \\
(95 \% \mathrm{CI})\end{array}$ & $\mathrm{P}$ & $\begin{array}{l}\text { Adjusted }^{\mathrm{a}} \mathrm{HR} \\
(95 \% \mathrm{CI})\end{array}$ & $\mathrm{P}$ & Case & $\begin{array}{l}\text { Person } \\
\text { years }\end{array}$ & $\begin{array}{c}\text { Unadjusted HR } \\
(95 \% \mathrm{CI})\end{array}$ & $\mathrm{P}$ & $\begin{array}{l}\text { Adjusted }^{\mathrm{b}} \mathrm{HR} \\
(95 \% \mathrm{CI})\end{array}$ & $\mathrm{P}$ \\
\hline & & \multicolumn{6}{|c|}{ Diagnosed 2009 to 2015} & \multicolumn{6}{|c|}{ Diagnosed 2012 to 2015 (additionally adjusted for stage and grade) } \\
\hline \multicolumn{2}{|l|}{ Untreated } & 1105 & 22590 & 1.00 (ref. cat.) & & 1.00 (ref. cat.) & & 511 & 10837 & 1.00 (ref. cat.) & & 1.00 (ref. cat.) & \\
\hline \multirow[t]{2}{*}{ ADT: } & Current use & 1752 & 24196 & $1.54(1.42,1.66)$ & $<0.001$ & $1.33(1.23,1.44)$ & $<0.001$ & 857 & 11896 & $1.60(1.42,1.79)$ & $<0.001$ & $1.40(1.18,1.66)$ & $<0.001$ \\
\hline & Previous use & 608 & 12984 & $1.04(0.93,1.15)$ & 0.482 & $1.06(0.95,1.18)$ & 0.276 & 212 & 4666 & $1.05(0.89,1.24)$ & 0.579 & $1.17(0.96,1.43)$ & 0.117 \\
\hline \multirow[t]{2}{*}{ GnRH: } & Current use & 1550 & 21724 & $1.52(1.40,1.64)$ & $<0.001$ & $1.33(1.23,1.44)$ & $<0.001$ & 735 & 10529 & $1.55(1.37,1.74)$ & $<0.001$ & $1.41(1.18,1.67)$ & $<0.001$ \\
\hline & Previous use & 525 & 11299 & $1.03(0.92,1.15)$ & 0.609 & $1.07(0.96,1.19)$ & 0.246 & 185 & 4046 & $1.06(0.88,1.26)$ & 0.538 & $1.19(0.97,1.46)$ & 0.091 \\
\hline \multirow[t]{2}{*}{ Degaralex: } & Current use & 66 & 683 & $2.02(1.57,2.59)$ & $<0.001$ & $1.50(1.16,1.93)$ & 0.002 & 59 & 621 & $2.08(1.58,2.73)$ & $<0.001$ & $1.47(0.97,2.24)$ & 0.072 \\
\hline & Previous use & 5 & 43 & $2.50(1.04,6.01)$ & 0.041 & $1.60(0.66,3.85)$ & 0.299 & 5 & 36 & $3.15(1.31,7.62)$ & 0.011 & $1.97(0.73,5.33)$ & 0.182 \\
\hline \multirow[t]{2}{*}{ Bicalutamide } & : Current use & 61 & 962 & $1.34(1.03,1.74)$ & 0.027 & $1.04(0.80,1.35)$ & 0.778 & 27 & 398 & $1.50(1.01,2.21)$ & 0.042 & $1.22(0.78,1.90)$ & 0.378 \\
\hline & Previous use & 34 & 749 & $1.01(0.71,1.42)$ & 0.97 & $0.96(0.68,1.35)$ & 0.807 & 10 & 223 & $1.03(0.55,1.94)$ & 0.92 & $0.96(0.49,1.88)$ & 0.914 \\
\hline \multicolumn{14}{|c|}{ Other antiandrogen: } \\
\hline & Current use & 47 & 501 & $1.94(1.45,2.60)$ & $<0.001$ & $1.44(1.07,1.93)$ & 0.016 & 21 & 223 & $2.05(1.32,3.17)$ & 0.001 & $1.40(0.74,2.65)$ & 0.307 \\
\hline & Previous use & 70 & 1158 & $1.32(1.03,1.68)$ & 0.026 & $1.21(0.95,1.54)$ & 0.128 & 26 & 474 & $1.24(0.83,1.84)$ & 0.287 & $1.23(0.75,2.01)$ & 0.411 \\
\hline
\end{tabular}

${ }^{a}$ Model contains year of diagnosis, age, deprivation, comorbidities in the year before prostate cancer diagnosis (myocardial infarction, angina, cerebral vascular accident, heart failure, venous thromboembolism, arrhythmia, other cardiovascular disease, dementia, pulmonary disease, connective tissue disorder, peptic ulcer, liver disease, diabetes, diabetes complications, paraplegia, severe liver disease) and other medication use in the year before prostate cancer diagnosis (including separately aspirin, beta-blockers, ACEIs, ARBs, diuretics, statins, warfarin, digoxin, clopidogrel, dipyridamole, nitrate, insulin, sulfonylureas, metformin and othaer diabetic medications). ${ }^{\mathrm{b}}$ Adjusted models contain all terms in ${ }^{\mathrm{a}}$ as well as $\mathrm{t}$ stage, $\mathrm{n}$ stage, $\mathrm{m}$ stage and Gleason score. 


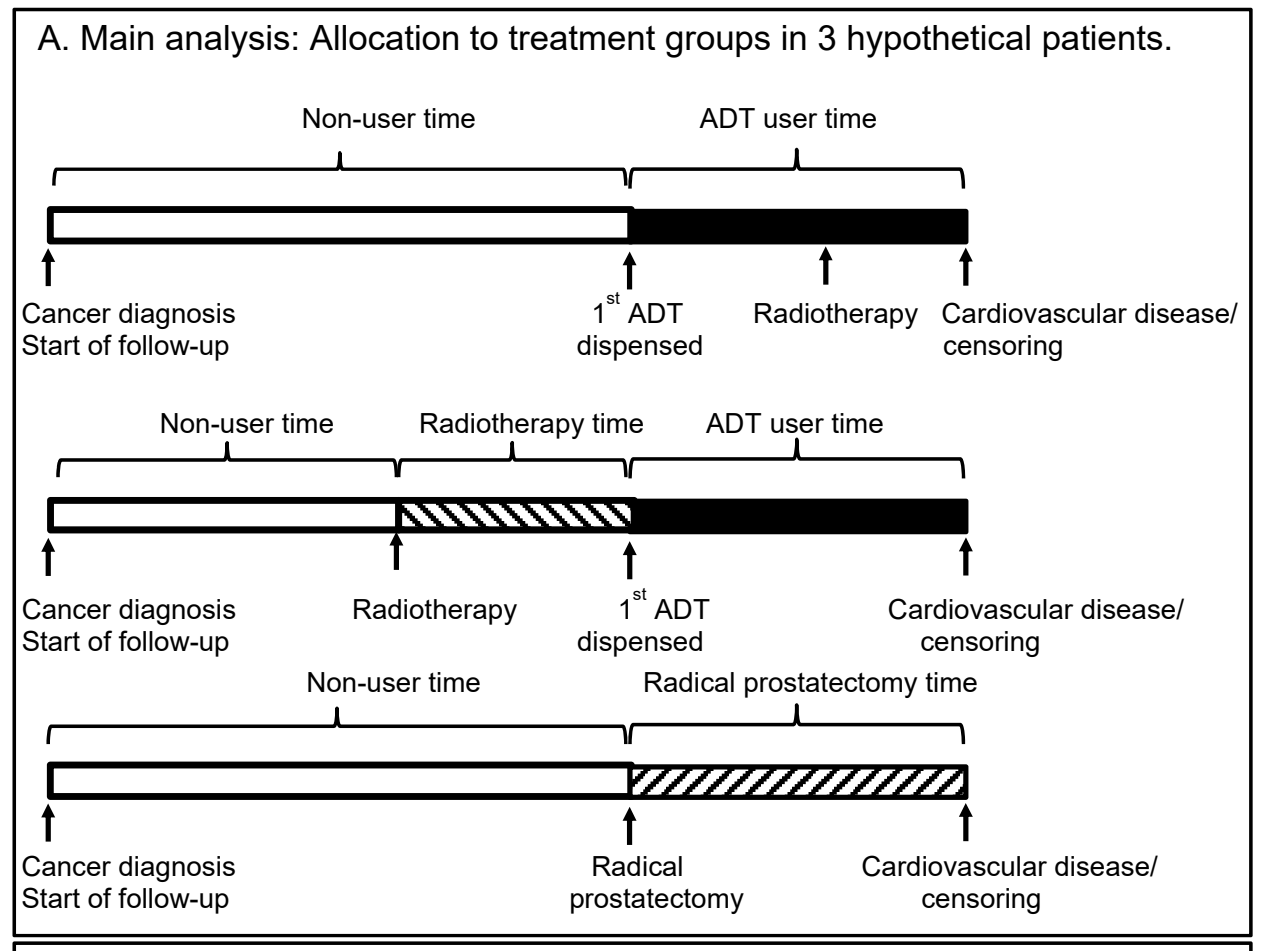

B. Analysis of ADT dose response.

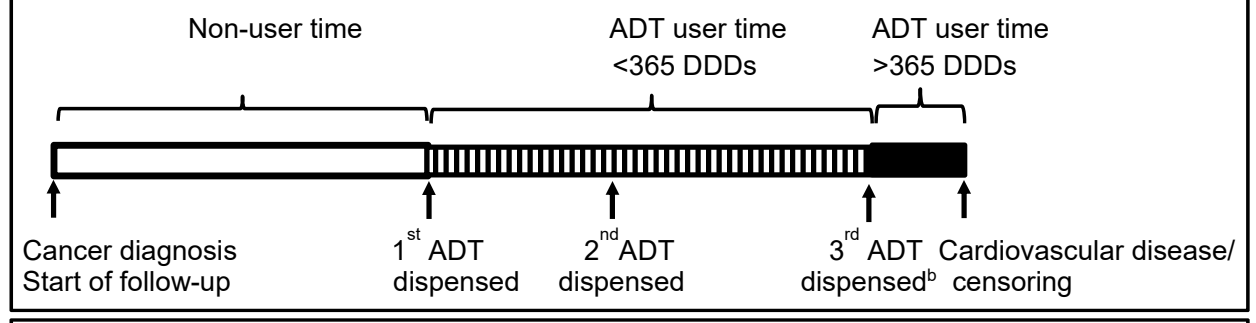

C. Analysis of current use of ADT (start/stop time-varying exposure) analysis.

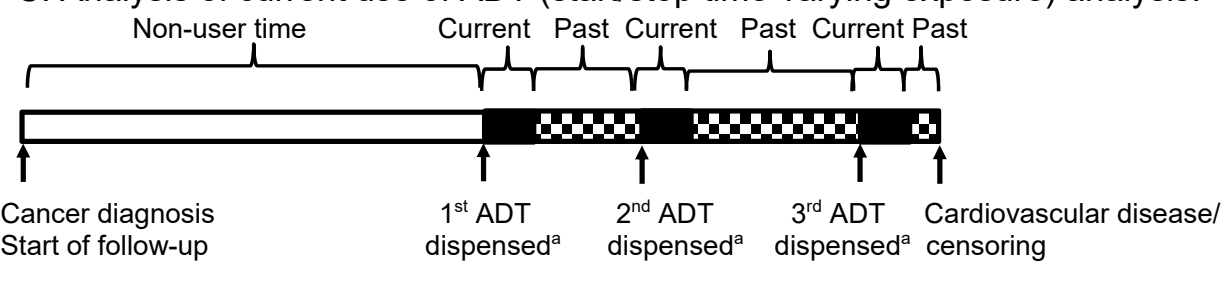

${ }^{\text {a}}$ Duration of user time depends on the number of DDD in the prescription plus a residual period as described.

${ }^{\mathrm{b}}$ Assuming on receipt of $3^{\text {rd }}$ dispensed ADT usage exceeds 365 DDDs. 
Figure 1. Figure illustrating the study design for the main analyses of ADT and secondary analysis of current use of ADT. 


\section{References}

1 Cornford P, Bellmunt J, Bolla M, Briers E, De Santis M, Gross T, et al. EAUESTRO-SIOG Guidelines on Prostate Cancer. Part II: Treatment of Relapsing, Metastatic, and Castration-Resistant Prostate Cancer. Eur Urol 2017;71:630-42. 2 Jones CU, Hunt D, McGowan DG, Amin MB, Chetner MP, Bruner DW, et al. Radiotherapy and Short-Term Androgen Deprivation for Localized Prostate Cancer. N Engl J Med 2011;365:107-18.

3 Mottet N, Bellmunt J, Bolla M, Briers E, Cumberbatch MG, De Santis M, et al. EAU-ESTRO-SIOG Guidelines on Prostate Cancer. Part 1: Screening, Diagnosis, and Local Treatment with Curative Intent. Eur Urol 2017;71:618-29.

4 Nguyen PL, Alibhai SMH, Basaria S, D’Amico A V., Kantoff PW, Keating NL, et al. Adverse effects of androgen deprivation therapy and strategies to mitigate them. Eur Urol 2015;67:825-36.

5 Zareba P, Duivenvoorden W, Leong DP, Pinthus JH. Androgen deprivation therapy and cardiovascular disease: What is the linking mechanism? Ther Adv Urol 2016;8:118-29.

6 Qiu Y, Yanase T, Hu H, Tanaka T, Nishi Y, Liu M, et al. Dihydrotestosterone suppresses foam cell formation and attenuates atherosclerosis development. Endocrinology 2010;151:3307-16. 7 Scailteux LM, Vincendeau S, Balusson F, Leclercq C, Happe A, Le Nautout B, et al. Androgen deprivation therapy and cardiovascular risk: No meaningful difference between GnRH antagonist and agonists - a nationwide population-based cohort study based on 2010-2013 French Health Insurance data. Eur J Cancer 2017;77:99-108. 8 Bosco C, Bosnyak Z, Malmberg A, Adolfsson J, Keating NL, Van Hemelrijck M. Quantifying Observational Evidence for Risk of Fatal and Nonfatal Cardiovascular Disease Following Androgen Deprivation Therapy for Prostate Cancer: A Metaanalysis. Eur Urol 2015;68:386-96.

9 Nguyen PL, Je Y, Schutz FAB, Hoffman KE, Hu JC, Parekh A, et al. Association of androgen deprivation therapy with cardiovascular death in patients with prostate cancer: A meta-analysis of randomized trials. JAMA 2011;306:2359-66.

10 Albertsen PC, Klotz L, Tombal B, Grady J, Olesen TK, Nilsson J. Cardiovascular morbidity associated with gonadotropin releasing hormone agonists and an antagonist. Eur Urol 2014;65:565-73.

11 Alvarez-Madrazo S, McTaggart S, Nangle C, Nicholson E, Bennie M. Data 
Resource Profile: The Scottish National Prescribing Information System (PIS). Int J Epidemiol 2016;45:714-715f.

12 Haque R, Ulcickasyood M, Xu X, Cassidy-Bushrow AE, Tsai HT, Keating NL, et al. Cardiovascular disease risk and androgen deprivation therapy in patients with localised prostate cancer: A prospective cohort study. Br J Cancer 2017;117:1233-40. 13 O'Farrell S, Garmo H, Holmberg L, Adolfsson J, Stattin P, Van Hemelrijck M. Risk and timing of cardiovascular disease after androgen-deprivation therapy in men with prostate cancer. J Clin Oncol 2015;33:1243-51.

14 Klil-Drori AJ, Yin H, Tagalakis V, Aprikian A, Azoulay L. Androgen Deprivation Therapy for Prostate Cancer and the Risk of Venous Thromboembolism. Eur Urol 2016;70:56-61.

15 World Health Organisation. World Health Organisation Collaborating Centre for Drug Statistics Methodology. Available at: https://www.whocc.no/atc/structure_and_principles/. Accessed Feb 2019. 16 Hussain S, Gunnell D, Donovan J, McPhail S, Hamdy F, Neal D, et al. Secular trends in prostate cancer mortality, incidence and treatment: England and Wales, 1975-2004. BJU Int 2008;101:547-55.

17 Sundararajan V, Henderson T, Perry C, Muggivan A, Quan H, Ghali WA. New ICD-10 version of the Charlson comorbidity index predicted in-hospital mortality. $\mathrm{J}$ Clin Epidemiol 2004;57:1288-94.

18 The Scottish Government. Scottish Index of Multiple Deprivation 2009: General Report. Edinburgh, UK: Scottish Government; 2009.

19 Van Hemelrijck M, Garmo H, Wigertz A, Nilsson P, Stattin P. Cohort profile update: The national prostate cancer register of Sweden and prostate cancer data baseA refined prostate cancer trajectory. Int J Epidemiol 2016;45:73-82.

20 National Institute for Health and Care Excellence. Prostate cancer:diagnosis and management (Clinical Guideline 175). Available at https://www.nice.org.uk/guidance/cg175. 2014. Accessed Feb 2019. 21 Lapi F, Azoulay L, Niazi MT, Yin H, Benayoun S, Suissa S. Androgen deprivation therapy and risk of acute kidney injury in patients with prostate cancer. JAMA 2013;310:289-96.

$22 \mathrm{Ng}$ HS, Koczwara B, Roder D, Vitry A. Development of comorbidities in men with prostate cancer treated with androgen deprivation therapy: an Australian population-based cohort study. Prostate Cancer Prostatic Dis 2018:1-8. 
23 Slovin SF, Melloni C, Mansor-Lefebvre S, Neijber AMR. A multicenter, randomized, controlled trial comparing the occurrence of major adverse cardiovascular events (MACEs) in patients (pts) with prostate cancer (pc) and cardiovascular disease (CVD) receiving degarelix (GnRH receptor antagonist) or leuprolide (GnRH receptor agonist). J Clin Oncol 2018;36:5101.

24 Wadhwa VK, Weston R, Parr NJ. Bicalutamide monotherapy preserves bone mineral density, muscle strength and has significant health-related quality of life benefits for osteoporotic men with prostate cancer. BJU Int 2011;107:1923-9. 25 Iversen P, Tyrrell CJ, Kaisary AV, Anderson JB, Van Poppel H, Tammela TLJ, et al. Bicalutamide monotherapy compared with castration in patients with nonmetastatic locally advanced prostate cancer: 6.3 years of followup. J Urol 2000;164:1579-82.

26 Beckmann K, Garmo H, Adolfsson J, Bosco C, Johansson E, Robinson D, et al. Androgen deprivation therapies and changes in comorbidity: A comparison of Gonadotropin-releasing Hormone Agonists and antiandrogen monotherapy as primary therapy in men with high-risk prostate cancer. Eur Urol 2018; doi:

10.1016/j.eururo.2018.11.022.

27 Information Service Division Scotland. Cancer incidence and prevalence in Scotland. Available at https://www.isdscotland.org/HealthTopics/Cancer/Publications/2019-04-30/2019-04-30-Cancer-Incidence-Report.pdf. Accessed Feb 2019.

28 Information Service Division Scotland. Assessment of SMR01 Data 2010-2011, Scotland. Available at http://www.isdscotland.org/Health-Topics/Hospital-

Care/Publications/2012-05-08/Assessment-of-SMR01Data-2010-2011ScotlandReport.pdf. Accessed Feb 2019.

29 Grundmark B, Garmo H, Zethelius B, Stattin P, Lambe M, Holmberg L. Antiandrogen prescribing patterns, patient treatment adherence and influencing factors; results from the nationwide PCBaSe Sweden. Eur J Clin Pharmacol 2012;68:161930. 
eTable 1. Association between androgen deprivation therapy and cardiovascular events adjusting for age.

\begin{tabular}{|c|c|c|c|c|c|c|c|c|c|c|c|c|c|}
\hline & & Case & $\begin{array}{c}\text { Person } \\
\text { years }\end{array}$ & $\begin{array}{c}\text { Unadjusted HR } \\
(95 \% \mathrm{CI})\end{array}$ & $\mathrm{P}$ & $\begin{array}{l}\text { Age-adjusted }^{\mathrm{a}} \\
\text { HR }(95 \% \mathrm{CI})\end{array}$ & $\mathrm{P}$ & Case & $\begin{array}{c}\text { Person } \\
\text { years }\end{array}$ & $\begin{array}{c}\text { Unadjusted HR } \\
(95 \% \mathrm{CI})\end{array}$ & $\mathrm{P}$ & $\begin{array}{l}\text { Age-adjusted }^{\mathrm{a}} \\
\text { HR }(95 \% \mathrm{CI})\end{array}$ & $\mathrm{P}$ \\
\hline & & \multicolumn{6}{|c|}{ Diagnosed 2009 to 2015} & \multicolumn{6}{|c|}{ Diagnosed 2012 to 2015 (additionally adjusted for stage and grade) } \\
\hline \multicolumn{2}{|l|}{ Untreated } & 1105 & 22590 & 1.00 (ref. cat.) & & 1.00 (ref. cat.) & & 511 & 10837 & 1.00 (ref. cat.) & & 1.00 (ref. cat.) & \\
\hline \multicolumn{2}{|c|}{ Radical prostatectomy } & 253 & 9350 & $0.61(0.53,0.70)$ & $<0.001$ & $0.83(0.72,0.96)$ & 0.013 & 104 & 3836 & $0.63(0.51,0.78)$ & $<0.001$ & $0.84(0.67,1.05)$ & 0.121 \\
\hline \multicolumn{2}{|c|}{ Radiotherapy } & 135 & 4450 & $0.68(0.57,0.82)$ & $<0.001$ & $0.81(0.68,0.97)$ & 0.023 & 53 & 1929 & $0.65(0.49,0.86)$ & 0.003 & $0.75(0.56,0.99)$ & 0.045 \\
\hline \multirow[t]{2}{*}{ ADT: } & Any use & 2360 & 37180 & $1.38(1.28,1.49)$ & $<0.001$ & $1.26(1.17,1.35)$ & $<0.001$ & 1069 & 16562 & $1.46(1.31,1.64)$ & $<0.001$ & $1.32(1.18,1.47)$ & $<0.001$ \\
\hline & 365+ DDDs & 1381 & 20685 & $1.56(1.43,1.70)$ & $<0.001$ & $1.38(1.26,1.50)$ & $<0.001$ & 576 & 8303 & $1.73(1.51,1.98)$ & $<0.001$ & $1.52(1.33,1.74)$ & $<0.001$ \\
\hline \multirow[t]{3}{*}{ GnRH: } & Any use & 2075 & 33023 & $1.37(1.27,1.48)$ & $<0.001$ & $1.25(1.15,1.35)$ & $<0.001$ & 920 & 14575 & $1.43(1.28,1.60)$ & $<0.001$ & $1.29(1.15,1.45)$ & $<0.001$ \\
\hline & 0-365 DDDs & 990 & 16606 & $1.24(1.14,1.36)$ & $<0.001$ & $1.17(1.07,1.28)$ & 0.001 & 487 & 8101 & $1.31(1.15,1.49)$ & $<0.001$ & $1.21(1.06,1.37)$ & 0.005 \\
\hline & $365+$ DDDs & 1085 & 16417 & $1.55(1.41,1.69)$ & $<0.001$ & $1.35(1.23,1.48)$ & $<0.001$ & 433 & 6473 & $1.65(1.43,1.90)$ & $<0.001$ & $1.43(1.24,1.66)$ & $<0.001$ \\
\hline \multirow[t]{3}{*}{ Degaralex: } & Any use & 71 & 726 & $2.01(1.58,2.56)$ & $<0.001$ & $1.73(1.36,2.20)$ & $<0.001$ & 64 & 657 & $2.12(1.63,2.76)$ & $<0.001$ & $1.83(1.41,2.38)$ & $<0.001$ \\
\hline & 0-365 DDDs & 41 & 393 & $1.98(1.45,2.71)$ & $<0.001$ & $1.73(1.27,2.37)$ & 0.001 & 37 & 361 & $2.05(1.47,2.88)$ & $<0.001$ & $1.80(1.29,2.52)$ & 0.001 \\
\hline & $365+$ DDDs & 30 & 333 & $2.01(1.39,2.89)$ & $<0.001$ & $1.71(1.19,2.46)$ & 0.004 & 27 & 296 & $2.20(1.49,3.26)$ & $<0.001$ & $1.88(1.27,2.78)$ & 0.002 \\
\hline \multirow[t]{3}{*}{ Bicalutamide: } & Any use & 95 & 1711 & $1.22(0.98,1.50)$ & 0.07 & $1.14(0.93,1.41)$ & 0.214 & 37 & 620 & $1.35(0.97,1.89)$ & 0.079 & $1.25(0.90,1.75)$ & 0.185 \\
\hline & 0-365 DDDs & 33 & 539 & $1.24(0.88,1.76)$ & 0.219 & $1.19(0.84,1.68)$ & 0.337 & 15 & 250 & $1.28(0.76,2.14)$ & 0.35 & $1.21(0.73,2.03)$ & 0.458 \\
\hline & $365+$ DDDs & 62 & 1172 & $1.22(0.94,1.58)$ & 0.13 & $1.13(0.88,1.47)$ & 0.34 & 22 & 370 & $1.44(0.93,2.21)$ & 0.098 & $1.31(0.85,2.01)$ & 0.225 \\
\hline \multicolumn{14}{|c|}{ Other antiandrogen: } \\
\hline & Any use & 117 & 1659 & $1.53(1.26,1.85)$ & $<0.001$ & $1.34(1.10,1.62)$ & 0.003 & 47 & 697 & $1.52(1.13,2.06)$ & 0.006 & $1.36(1.01,1.84)$ & 0.044 \\
\hline & 0-365 DDDs & 103 & 1520 & $1.47(1.20,1.80)$ & $<0.001$ & $1.29(1.05,1.58)$ & 0.014 & 42 & 667 & $1.43(1.04,1.97)$ & 0.026 & $1.29(0.94,1.77)$ & 0.111 \\
\hline & $365+$ DDDs & 14 & 139 & $2.43(1.43,4.12)$ & 0.001 & $1.98(1.16,3.36)$ & 0.012 & 5 & 30 & $4.21(1.74,10.18)$ & 0.001 & $2.99(1.23,7.25)$ & 0.015 \\
\hline
\end{tabular}

${ }^{\text {a}}$ Model contains age. 
eTable 2. Association between androgen deprivation therapy and cardiovascular disease based upon incidence.

\begin{tabular}{|c|c|c|c|c|c|c|c|c|c|c|c|c|c|}
\hline & & Case & $\begin{array}{c}\text { Person } \\
\text { years }\end{array}$ & $\begin{array}{c}\text { Unadjusted HR } \\
(95 \% \mathrm{CI})\end{array}$ & $\mathrm{P}$ & $\begin{array}{c}\text { Adjusted }^{\mathrm{a}} \mathrm{HR} \\
(95 \% \mathrm{CI})\end{array}$ & $\mathrm{P}$ & Case & $\begin{array}{c}\text { Person } \\
\text { years }\end{array}$ & $\begin{array}{c}\text { Unadjusted HR } \\
(95 \% \mathrm{CI})\end{array}$ & $\mathrm{P}$ & $\begin{array}{c}\text { Adjusted }^{\mathrm{b}} \mathrm{HR}(95 \% \\
\mathrm{CI})\end{array}$ & $\mathrm{P}$ \\
\hline \multirow{2}{*}{\multicolumn{2}{|c|}{ Untreated }} & \multicolumn{6}{|c|}{ Diagnosed 2009 to 2015} & \multicolumn{6}{|c|}{ Diagnosed 2012 to 2015 (additionally adjusted for stage and grade) } \\
\hline & & 990 & 22590 & 1.00 (ref. cat.) & & 1.00 (ref. cat.) & & 462 & 10837 & 1.00 (ref. cat.) & & 1.00 (ref. cat.) & \\
\hline \multicolumn{2}{|c|}{ Radical prostatectomy } & 243 & 9350 & $0.65(0.56,0.75)$ & $<0.001$ & $0.94(0.81,1.09)$ & 0.412 & 99 & 3836 & $0.66(0.53,0.82)$ & $<0.001$ & $0.96(0.75,1.23)$ & 0.75 \\
\hline \multicolumn{2}{|l|}{ Radiotherapy } & 129 & 4450 & $0.73(0.61,0.88)$ & 0.001 & $0.87(0.72,1.05)$ & 0.14 & 51 & 1929 & $0.68(0.51,0.92)$ & 0.011 & $0.85(0.63,1.15)$ & 0.293 \\
\hline \multirow[t]{3}{*}{ ADT: } & Any use & 2082 & 37180 & $1.36(1.26,1.48)$ & $<0.001$ & $1.25(1.15,1.35)$ & $<0.001$ & 951 & 16562 & $1.43(1.27,1.61)$ & $<0.001$ & $1.33(1.13,1.56)$ & 0.001 \\
\hline & 0-365 DDDs & 863 & 16495 & $1.21(1.10,1.33)$ & $<0.001$ & $1.14(1.04,1.25)$ & 0.007 & 436 & 8259 & $1.25(1.09,1.43)$ & 0.001 & $1.22(1.02,1.45)$ & 0.025 \\
\hline & 365+ DDDs & 1219 & 20685 & $1.54(1.41,1.69)$ & $<0.001$ & $1.36(1.24,1.50)$ & $<0.001$ & 515 & 8303 & $1.69(1.47,1.95)$ & $<0.001$ & $1.61(1.32,1.97)$ & $<0.001$ \\
\hline \multirow[t]{3}{*}{ GnRH: } & Any use & 1845 & 33023 & $1.36(1.26,1.48)$ & $<0.001$ & $1.26(1.16,1.37)$ & $<0.001$ & 830 & 14575 & $1.42(1.26,1.60)$ & $<0.001$ & $1.35(1.15,1.60)$ & $<0.001$ \\
\hline & 0-365 DDDs & 888 & 16606 & $1.25(1.13,1.37)$ & $<0.001$ & $1.19(1.08,1.30)$ & $<0.001$ & 441 & 8101 & $1.30(1.14,1.49)$ & $<0.001$ & $1.28(1.08,1.53)$ & 0.005 \\
\hline & 365+ DDDs & 957 & 16417 & $1.52(1.38,1.68)$ & $<0.001$ & $1.36(1.24,1.50)$ & $<0.001$ & 389 & 6473 & $1.61(1.39,1.88)$ & $<0.001$ & $1.59(1.29,1.97)$ & $<0.001$ \\
\hline \multirow[t]{3}{*}{ Degarelix: } & Any use & 60 & 726 & $1.89(1.46,2.46)$ & $<0.001$ & $1.44(1.10,1.88)$ & 0.007 & 55 & 657 & $2.00(1.51,2.65)$ & $<0.001$ & $1.43(0.94,2.17)$ & 0.094 \\
\hline & 0-365 DDDs & 35 & 393 & $1.89(1.35,2.65)$ & $<0.001$ & $1.43(1.01,2.01)$ & 0.042 & 32 & 361 & $1.96(1.37,2.82)$ & $<0.001$ & $1.29(0.74,2.25)$ & 0.362 \\
\hline & 365+ DDDs & 25 & 333 & $1.86(1.25,2.78)$ & 0.002 & $1.45(0.97,2.17)$ & 0.071 & 23 & 296 & $2.04(1.34,3.12)$ & 0.001 & $1.77(0.99,3.18)$ & 0.054 \\
\hline \multirow[t]{3}{*}{ Bicalutamide: } & : Any use & 77 & 1711 & $1.10(0.87,1.39)$ & 0.418 & $0.92(0.73,1.17)$ & 0.51 & 28 & 620 & $1.12(0.76,1.65)$ & 0.557 & $1.04(0.69,1.57)$ & 0.852 \\
\hline & 0-365 DDDs & 27 & 539 & $1.14(0.78,1.67)$ & 0.512 & $0.99(0.68,1.45)$ & 0.963 & 11 & 250 & $1.03(0.57,1.88)$ & 0.917 & $0.94(0.48,1.84)$ & 0.865 \\
\hline & 365+ DDDs & 50 & 1172 & $1.10(0.82,1.46)$ & 0.521 & $0.90(0.68,1.20)$ & 0.476 & 17 & 370 & $1.21(0.75,1.98)$ & 0.437 & $1.15(0.70,1.91)$ & 0.574 \\
\hline \multicolumn{14}{|c|}{ Other antiandrogen: } \\
\hline & Any use & 98 & 1659 & $1.43(1.16,1.76)$ & 0.001 & $1.24(1.01,1.53)$ & 0.044 & 37 & 697 & $1.31(0.94,1.84)$ & 0.112 & $1.21(0.78,1.86)$ & 0.395 \\
\hline & 0-365 DDDs & 88 & 1520 & $1.40(1.13,1.75)$ & 0.003 & $1.24(1.00,1.55)$ & 0.052 & 32 & 667 & $1.20(0.83,1.72)$ & 0.328 & $1.16(0.73,1.83)$ & 0.531 \\
\hline & $365+$ DDDs & 10 & 139 & $1.93(1.04,3.61)$ & 0.038 & $1.29(0.69,2.42)$ & 0.432 & 5 & 30 & $4.58(1.89,11.09)$ & 0.001 & $3.54(1.11,11.28)$ & 0.032 \\
\hline
\end{tabular}

${ }^{a}$ Model contains year of diagnosis, age, deprivation, comorbidities in year before prostate cancer diagnosis (myocardial infarction, angina, cerebral vascular accident, heart failure, venous thromboembolism, arrhythmia, other cardiovascular disease, dementia, pulmonary disease, connective tissue disorder, peptic ulcer, liver disease, diabetes, diabetes complications, paraplegia, severe liver disease) and other medication use in the year before prostate cancer diagnosis (including aspirin, beta-blockers, ACEIs,

ARBs, diuretics, statins, warfarin, digoxin, clopidogrel, dipyridamole, nitrate, insulin, sulfonylureas, metformin and othaer diabetic medications). ${ }^{b}$ Adjusted models contain all terms in ${ }^{a}$ as well as $t$ stage, $n$ stage, $m$ stage and gleason score. 
eTable 3. Association between androgen deprivation therapy and cardiovascular disease based upon mortality.

\begin{tabular}{|c|c|c|c|c|c|c|c|c|c|c|c|c|c|}
\hline & & Case & $\begin{array}{l}\text { Person } \\
\text { years }\end{array}$ & $\begin{array}{c}\text { Unadjusted HR } \\
(95 \% \mathrm{CI})\end{array}$ & $\mathrm{P}$ & $\begin{array}{l}\text { Adjusted }^{\mathrm{a}} \mathrm{HR} \\
(95 \% \mathrm{CI})\end{array}$ & $\mathrm{P}$ & Case & $\begin{array}{l}\text { Person } \\
\text { years }\end{array}$ & $\begin{array}{l}\text { Unadjusted HR } \\
(95 \% \mathrm{CI})\end{array}$ & $\mathrm{P}$ & $\begin{array}{c}\text { Adjusted }^{\mathrm{b}} \mathrm{HR} \\
(95 \% \mathrm{CI})\end{array}$ & $\mathrm{P}$ \\
\hline & & \multicolumn{6}{|c|}{ Diagnosed 2009 to 2015} & \multicolumn{6}{|c|}{ Diagnosed 2012 to 2015 (additionally adjusted for stage and grade) } \\
\hline \multicolumn{2}{|l|}{ Untreated } & 221 & 24580 & 1.00 (ref. cat.) & & & & 95 & 11509 & 1.00 (ref. cat.) & & & \\
\hline \multicolumn{2}{|c|}{ Radical prostatectomy } & 16 & 10087 & $0.18(0.11,0.30)$ & $<0.001$ & $0.62(0.37,1.05)$ & 0.074 & 5 & 4033 & $0.16(0.07,0.41)$ & $<0.001$ & $0.56(0.21,1.48)$ & 0.241 \\
\hline \multicolumn{2}{|c|}{ Radiotherapy } & 10 & 4804 & $0.24(0.13,0.45)$ & $<0.001$ & $0.47(0.25,0.88)$ & 0.019 & $<5$ & 2036 & $0.26(0.10,0.72)$ & 0.009 & $0.38(0.11,1.26)$ & 0.113 \\
\hline \multirow[t]{3}{*}{ ADT: } & Any use & 571 & 41954 & $1.57(1.33,1.85)$ & $<0.001$ & $1.35(1.15,1.59)$ & $<0.001$ & 227 & 18175 & $1.65(1.27,2.13)$ & $<0.001$ & $1.04(0.66,1.65)$ & 0.864 \\
\hline & 0-365 DDDs & 197 & 17922 & $1.26(1.03,1.54)$ & 0.023 & $1.23(1.01,1.50)$ & 0.043 & 94 & 8800 & $1.37(1.02,1.86)$ & 0.038 & $0.85(0.50,1.42)$ & 0.531 \\
\hline & 365+ DDDs & 374 & 24032 & $1.87(1.56,2.24)$ & $<0.001$ & $1.45(1.21,1.74)$ & $<0.001$ & 133 & 9376 & $2.01(1.49,2.71)$ & $<0.001$ & $1.32(0.78,2.22)$ & 0.3 \\
\hline \multirow[t]{3}{*}{ GnRH: } & Any use & 491 & 37285 & $1.51(1.28,1.79)$ & $<0.001$ & $1.34(1.13,1.58)$ & 0.001 & 185 & 15971 & $1.52(1.17,1.98)$ & 0.002 & $1.01(0.63,1.61)$ & 0.964 \\
\hline & $0-365$ DDDs & 185 & 18097 & $1.16(0.95,1.42)$ & 0.143 & $1.16(0.95,1.43)$ & 0.146 & 86 & 8653 & $1.28(0.94,1.75)$ & 0.112 & $0.98(0.59,1.63)$ & 0.947 \\
\hline & 365+ DDDs & 306 & 19188 & $1.94(1.60,2.34)$ & $<0.001$ & $1.50(1.24,1.81)$ & $<0.001$ & 99 & 7318 & $1.90(1.38,2.62)$ & $<0.001$ & $1.08(0.62,1.86)$ & 0.792 \\
\hline \multirow[t]{3}{*}{ Degarelix: } & Any use & 20 & 832 & $2.81(1.77,4.46)$ & $<0.001$ & $1.71(1.07,2.74)$ & 0.026 & 17 & 753 & $2.97(1.76,5.02)$ & $<0.001$ & $1.58(0.61,4.10)$ & 0.346 \\
\hline & 0-365 DDDs & 9 & 440 & $2.24(1.14,4.38)$ & 0.019 & $1.41(0.72,2.78)$ & 0.32 & 8 & 399 & $2.47(1.19,5.15)$ & 0.015 & $1.15(0.26,5.03)$ & 0.858 \\
\hline & 365+ DDDs & 11 & 392 & $3.37(1.83,6.22)$ & $<0.001$ & $2.05(1.10,3.82)$ & 0.025 & 9 & 354 & $3.56(1.77,7.16)$ & $<0.001$ & $2.04(0.66,6.32)$ & 0.218 \\
\hline \multirow[t]{3}{*}{ Bicalutamide: } & Any use & 28 & 1908 & $1.68(1.13,2.50)$ & 0.01 & $1.39(0.93,2.06)$ & 0.107 & 11 & 676 & $2.14(1.14,4.03)$ & 0.018 & $1.17(0.47,2.94)$ & 0.731 \\
\hline & 0-365 DDDs & 11 & 585 & $2.13(1.16,3.92)$ & 0.014 & $1.85(1.00,3.41)$ & 0.049 & 5 & 260 & $2.49(1.01,6.15)$ & 0.047 & $0.76(0.10,5.61)$ & 0.788 \\
\hline & 365+ DDDs & 17 & 1323 & $1.53(0.93,2.52)$ & 0.094 & $1.21(0.73,2.00)$ & 0.452 & 6 & 416 & $1.99(0.86,4.58)$ & 0.107 & $1.36(0.49,3.72)$ & 0.553 \\
\hline \multicolumn{14}{|c|}{ Other antiandrogen: } \\
\hline & Any use & 31 & 1864 & $1.92(1.32,2.80)$ & 0.001 & $1.41(0.96,2.06)$ & 0.077 & 13 & 763 & $2.26(1.26,4.06)$ & 0.007 & $1.13(0.42,3.07)$ & 0.811 \\
\hline & 0-365 DDDs & 25 & 1687 & $1.74(1.15,2.64)$ & 0.009 & $1.31(0.86,1.99)$ & 0.202 & $9-13$ & 724 & $2.24(1.22,4.11)$ & 0.009 & $1.28(0.47,3.50)$ & 0.632 \\
\hline & 365+ DDDs & 6 & 177 & $4.11(1.82,9.29)$ & 0.001 & $2.22(0.97,5.06)$ & 0.058 & $<5$ & 38 & $3.66(0.51,26.44)$ & 0.198 & & \\
\hline
\end{tabular}

${ }^{\mathrm{a}}$ Model contains year of diagnosis, age, deprivation, comorbidities in year before prostate cancer diagnosis (myocardial infarction, angina, cerebral vascular accident, heart failure, venous thromboembolism, arrhythmia, other cardiovascular disease, dementia, pulmonary disease, connective tissue disorder, peptic ulcer, liver disease, diabetes, diabetes complications, paraplegia, severe liver disease) and other medication use in the year before prostate cancer diagnosis (including aspirin, beta-blockers, ACEIs,

ARBs, diuretics, statins, warfarin, digoxin, clopidogrel, dipyridamole, nitrate, insulin, sulfonylureas, metformin and othaer diabetic medications). ${ }^{b}$ Adjusted models contain all terms in ${ }^{a}$ as well as $t$ stage, $n$ stage, $m$ stage and gleason score. 
eTable 4. Association between androgen deprivation therapy and type of cardiovascular events (restricted to patients diagnosed 2012 to 2015 ).

\begin{tabular}{|c|c|c|c|c|c|c|}
\hline & Case & $\begin{array}{l}\text { Person } \\
\text { years }\end{array}$ & $\begin{array}{c}\text { Unadjusted HR } \\
(95 \% \mathrm{CI})\end{array}$ & $\mathrm{P}$ & $\begin{array}{c}\text { Adjusted }^{\mathrm{a}} \mathrm{HR} \\
(95 \% \mathrm{CI})\end{array}$ & $\mathrm{P}$ \\
\hline \multicolumn{7}{|c|}{ Angina } \\
\hline Untreated & 135 & 11315 & 1.00 (ref. cat.) & & & \\
\hline $\mathrm{ADT}$ & 240 & 17833 & $1.08(0.86,1.34)$ & 0.516 & $1.13(0.84,1.53)$ & 0.427 \\
\hline GnRH & 205 & 15666 & $1.04(0.83,1.31)$ & 0.712 & $1.13(0.83,1.53)$ & 0.431 \\
\hline Degarelix & 13 & 740 & $1.42(0.80,2.52)$ & 0.227 & $1.01(0.45,2.27)$ & 0.975 \\
\hline Bicalutamide & 13 & 659 & $1.58(0.89,2.80)$ & 0.118 & $1.33(0.70,2.55)$ & 0.383 \\
\hline Other antiandrogen & 9 & 755 & $0.96(0.49,1.88)$ & 0.894 & $0.74(0.27,2.07)$ & 0.568 \\
\hline \multicolumn{7}{|c|}{ Myocardial infarction } \\
\hline Untreated & 91 & 11419 & 1.00 (ref. cat.) & & & \\
\hline ADT & 183 & 17973 & $1.39(1.06,1.81)$ & 0.016 & $1.32(0.89,1.96)$ & 0.163 \\
\hline GnRH & 153 & 15806 & $1.32(1.00,1.73)$ & 0.049 & $1.27(0.85,1.90)$ & 0.239 \\
\hline Degarelix & 12 & 742 & $2.11(1.15,3.87)$ & 0.016 & $1.55(0.62,3.84)$ & 0.349 \\
\hline Bicalutamide & 10 & 665 & $2.05(1.06,3.95)$ & 0.033 & $1.91(0.90,4.05)$ & 0.09 \\
\hline Other antiandrogen & 8 & 747 & $1.46(0.70,3.02)$ & 0.311 & $1.22(0.42,3.51)$ & 0.713 \\
\hline \multicolumn{7}{|c|}{ Arrhythmia } \\
\hline Untreated & 78 & 11419 & 1.00 (ref. cat.) & & & \\
\hline $\mathrm{ADT}$ & 185 & 17879 & $1.56(1.18,2.07)$ & 0.002 & $1.38(0.94,2.03)$ & 0.102 \\
\hline $\mathrm{GnRH}$ & 158 & 15713 & $1.51(1.14,2.02)$ & 0.004 & $1.40(0.94,2.07)$ & 0.096 \\
\hline Degarelix & 13 & 733 & $2.68(1.48,4.85)$ & 0.001 & $1.72(0.70,4.27)$ & 0.239 \\
\hline Bicalutamide & 6 & 670 & $1.35(0.58,3.10)$ & 0.485 & $1.06(0.41,2.69)$ & 0.91 \\
\hline Other antiandrogen & 8 & 750 & $1.62(0.78,3.36)$ & 0.198 & $1.41(0.54,3.66)$ & 0.485 \\
\hline \multicolumn{7}{|c|}{ Heart failure } \\
\hline Untreated & 46 & 11456 & 1.00 (ref. cat.) & & & \\
\hline ADT & 112 & 18028 & $1.67(1.16,2.39)$ & 0.006 & $1.25(0.68,2.30)$ & 0.467 \\
\hline $\mathrm{GnRH}$ & 96 & 15845 & $1.63(1.12,2.36)$ & 0.01 & $1.22(0.65,2.27)$ & 0.538 \\
\hline Degarelix & 8 & 743 & $2.66(1.25,5.67)$ & 0.011 & $0.88(0.19,4.07)$ & 0.872 \\
\hline Bicalutamide & $<5$ & 668 & $2.02(0.80,5.12)$ & 0.139 & $2.43(0.88,6.71)$ & 0.087 \\
\hline Other antiandrogen & $<5$ & 758 & $1.06(0.33,3.44)$ & 0.917 & $0.51(0.06,3.99)$ & 0.519 \\
\hline \multicolumn{7}{|c|}{ Stroke } \\
\hline Untreated & 99 & 11381 & 1.00 (ref. cat.) & & 1.00 (ref. cat.) & \\
\hline ADT & 185 & 17924 & $1.18(0.92,1.53)$ & 0.197 & $0.99(0.67,1.44)$ & 0.946 \\
\hline $\mathrm{GnRH}$ & 165 & 15755 & $1.20(0.93,1.56)$ & 0.164 & $1.02(0.70,1.50)$ & 0.912 \\
\hline Degarelix & $<5$ & 739 & $0.63(0.23,1.70)$ & 0.358 & $0.21(0.03,1.59)$ & 0.131 \\
\hline Bicalutamide & $<5$ & 670 & $0.86(0.35,2.11)$ & 0.734 & $0.60(0.18,1.93)$ & 0.388 \\
\hline Other antiandrogen & 10 & 748 & $1.53(0.79,2.94)$ & 0.203 & $1.44(0.62,3.33)$ & 0.395 \\
\hline \multicolumn{7}{|c|}{ Venous thromboembolism } \\
\hline Untreated & 47 & 11460 & 1.00 (ref. cat.) & & 1.00 (ref. cat.) & \\
\hline ADT & 119 & 17993 & $2.05(1.43,2.96)$ & $<0.001$ & $1.26(0.76,2.09)$ & 0.378 \\
\hline $\mathrm{GnRH}$ & 102 & 15816 & $2.00(1.38,2.91)$ & $<0.001$ & $1.33(0.79,2.21)$ & 0.281 \\
\hline Degarelix & 9 & 735 & $3.44(1.67,7.07)$ & 0.001 & $0.79(0.18,3.49)$ & 0.753 \\
\hline Bicalutamide & $<5$ & 675 & $0.45(0.06,3.30)$ & 0.434 & $0.37(0.05,2.77)$ & 0.331 \\
\hline Other antiandrogen & 7 & 754 & $2.81(1.26,6.27)$ & 0.012 & $1.40(0.47,4.23)$ & 0.547 \\
\hline \multicolumn{7}{|c|}{ Other CVD events } \\
\hline Untreated & 137 & 11331 & 1.00 (ref. cat.) & & 1.00 (ref. cat.) & \\
\hline ADT & 319 & 17693 & $1.55(1.25,1.91)$ & $<0.001$ & $1.68(1.24,2.27)$ & 0.001 \\
\hline GnRH & 282 & 15538 & $1.55(1.25,1.93)$ & $<0.001$ & $1.73(1.28,2.35)$ & $<0.001$ \\
\hline Degarelix & 20 & 730 & $2.32(1.45,3.72)$ & $<0.001$ & $2.60(1.35,4.99)$ & 0.004 \\
\hline Bicalutamide & 5 & 663 & $0.64(0.26,1.58)$ & 0.336 & $0.73(0.29,1.82)$ & 0.5 \\
\hline Other antiandrogen & 12 & 749 & $1.37(0.75,2.47)$ & 0.303 & $1.64(0.80,3.36)$ & 0.179 \\
\hline
\end{tabular}


${ }^{a}$ Model contains $\mathrm{t}$ stage, $\mathrm{n}$ stage, $\mathrm{m}$ stage, gleason score, year of diagnosis, age, deprivation, comorbidities in the year before prostate cancer diagnosis (myocardial infarction, angina, cerebral vascular accident, heart failure, venous thromboembolism, arrhythmia, other cardiovascular disease, dementia, pulmonary disease, connective tissue disorder, peptic ulcer, liver disease, diabetes, diabetes complications, paraplegia, severe liver disease) and other medication use in the year before prostate cancer diagnosis (including aspirin, beta-blockers, ACEIs, ARBs, diuretics, statins, warfarin, digoxin, clopidogrel, dipyridamole, nitrate, insulin, sulfonylureas, metformin and othaer diabetic medications). 
eTable 5. Association between androgen deprivation therapy and cardiovascular events stratifying by stage.

\begin{tabular}{|c|c|c|c|c|c|c|}
\hline & Case & $\begin{array}{c}\text { Person } \\
\text { years }\end{array}$ & $\begin{array}{c}\text { Unadjusted HR } \\
(95 \% \mathrm{CI})\end{array}$ & $\mathrm{P}$ & $\begin{array}{c}\text { Adjusted }{ }^{\mathrm{b}} \mathrm{HR} \\
(95 \% \mathrm{CI})\end{array}$ & $\mathrm{P}$ \\
\hline \multicolumn{7}{|c|}{ Diagnosed 2012 to 2015 (additionally adjusted for stage and Gleason) } \\
\hline \multicolumn{7}{|c|}{ No metastases (M stage 0 ) } \\
\hline Untreated & 287 & 6743 & 1.00 (ref. cat.) & & 1.00 (ref. cat.) & \\
\hline ADT & 588 & 10159 & $1.37(1.18,1.59)$ & $<0.001$ & $1.39(1.15,1.68)$ & 0.001 \\
\hline $\mathrm{GnRH}$ & 523 & 9238 & $1.34(1.15,1.55)$ & $<0.001$ & $1.40(1.15,1.70)$ & 0.001 \\
\hline Degaralex & 14 & 119 & $2.70(1.58,4.62)$ & $<0.001$ & $1.80(0.98,3.28)$ & 0.057 \\
\hline Bicalutamide & 30 & 420 & $1.68(1.15,2.45)$ & 0.007 & $1.32(0.87,2.00)$ & 0.192 \\
\hline Other antiandrogen & 21 & 380 & $1.30(0.84,2.04)$ & 0.241 & $1.16(0.69,1.96)$ & 0.584 \\
\hline \multicolumn{7}{|c|}{ Metastases (M stage 1) } \\
\hline Untreated & 52 & 465 & 1.00 (ref. cat.) & & 1.00 (ref. cat.) & \\
\hline ADT & 327 & 3948 & $0.86(0.59,1.25)$ & 0.425 & $1.94(0.79,4.75)$ & 0.148 \\
\hline GnRH & 268 & 3231 & $0.87(0.60,1.27)$ & 0.466 & $2.00(0.81,4.95)$ & 0.133 \\
\hline Degaralex & 40 & 470 & $0.88(0.55,1.40)$ & 0.585 & $1.73(0.61,4.92)$ & 0.301 \\
\hline Bicalutamide & $<5$ & 29 & $0.34(0.05,2.48)$ & 0.287 & & \\
\hline Other antiandrogen & 17 & 208 & $0.85(0.47,1.54)$ & 0.59 & $2.33(0.72,7.48)$ & 0.156 \\
\hline \multicolumn{7}{|c|}{ Low risk (T1N0M0 or T2N0M0) } \\
\hline Untreated & 197 & 5311 & 1.00 (ref. cat.) & & 1.00 (ref. cat.) & \\
\hline ADT & 233 & 4187 & $1.62(1.32,1.97)$ & $<0.001$ & $1.49(1.17,1.89)$ & 0.001 \\
\hline $\mathrm{GnRH}$ & 213 & 3790 & $1.63(1.33,2.00)$ & $<0.001$ & $1.54(1.21,1.96)$ & $<0.001$ \\
\hline Degaralex & $<5$ & 34 & $2.48(0.79,7.76)$ & 0.119 & $2.04(0.64,6.54)$ & 0.229 \\
\hline Bicalutamide & 13 & 222 & $1.69(0.96,2.97)$ & 0.069 & $1.20(0.66,2.21)$ & 0.551 \\
\hline Other antiandrogen & $<5$ & 139 & $0.83(0.31,2.23)$ & 0.711 & $0.73(0.27,2.00)$ & 0.538 \\
\hline
\end{tabular}

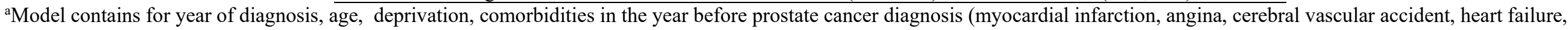

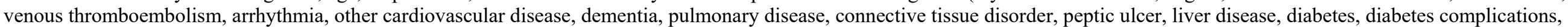

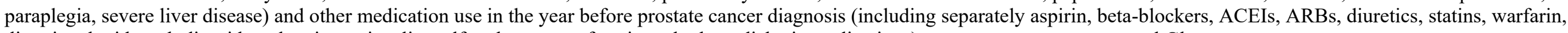
digoxin, clopidogrel, dipyridamole, nitrate, insulin, sulfonylureas, metformin and othaer diabetic medications), $\mathrm{t}$ stage, $\mathrm{n}$ stage, $\mathrm{m}$ stage and Gleason score. 
eTable 6. Association between androgen deprivation therapy and cardiovascular events stratifying by age.

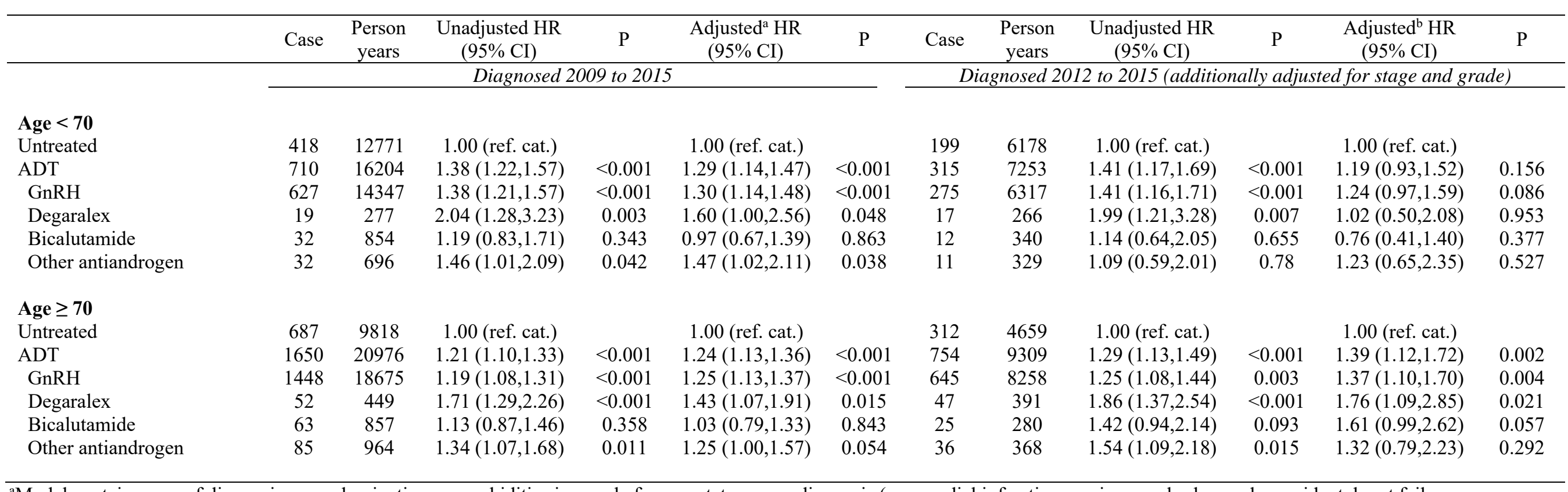

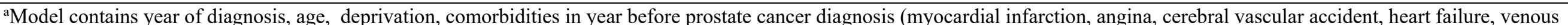

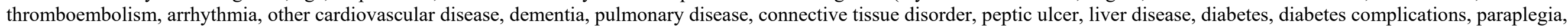

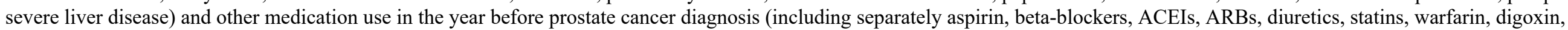

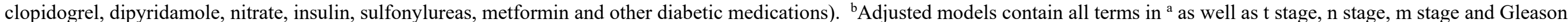
score. 
eTable 7. Association between androgen deprivation therapy and cardiovascular events, adjusting for stage and grade, treating stage $\mathrm{X}$ as missing.

\begin{tabular}{lcccccc}
\hline & Case & $\begin{array}{c}\text { Person } \\
\text { years }\end{array}$ & $\begin{array}{c}\text { Unadjusted HR } \\
(95 \% \mathrm{CI})\end{array}$ & $\mathrm{P}$ & $\begin{array}{c}\text { Adjusted } \mathrm{HR} \\
(95 \% \mathrm{CI})\end{array}$ & $\mathrm{P}$ \\
\hline & \multicolumn{7}{c}{ Diagnosed 2012 to 2015 (additionally adjusted for stage and grade) } \\
\cline { 2 - 7 } & 213 & 6210 & 1.00 (ref. cat.) & & 1.00 (ref. cat.) & \\
Untreated & 590 & 10843 & $1.60(1.35,1.88)$ & $<0.001$ & $1.48(1.21,1.80)$ & $<0.001$ \\
ADT & 528 & 9836 & $1.58(1.33,1.86)$ & $<0.001$ & $1.49(1.22,1.82)$ & $<0.001$ \\
GnRH & 18 & 208 & $2.47(1.52,4.00)$ & $<0.001$ & $1.74(1.05,2.90)$ & 0.033 \\
Degaralex & 24 & 393 & $1.80(1.18,2.75)$ & 0.007 & $1.29(0.83,2.01)$ & 0.248 \\
Bicalutamide & 19 & 400 & $1.40(0.87,2.24)$ & 0.165 & $1.32(0.81,2.15)$ & 0.269 \\
Other antiandrogen & & & & & &
\end{tabular}

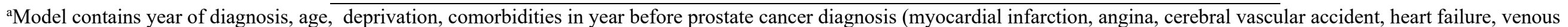

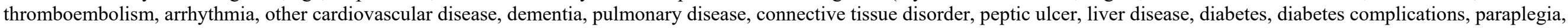

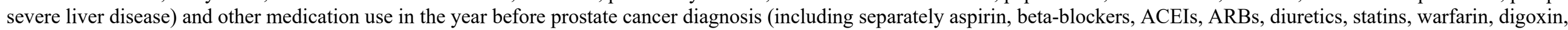
clopidogrel, dipyridamole, nitrate, insulin, sulfonylureas, metformin and other diabetic medications), $\mathrm{t}$ stage, $\mathrm{n}$ stage, $\mathrm{m}$ stage and Gleason score. 


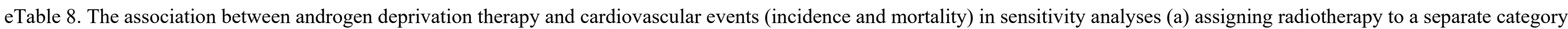
and (b) basing treatment solely upon initial treatment.

\begin{tabular}{|c|c|c|c|c|c|c|c|c|c|c|c|c|}
\hline & Case & $\begin{array}{c}\text { Person } \\
\text { years }\end{array}$ & $\begin{array}{c}\text { Unadjusted HR } \\
(95 \% \mathrm{CI})\end{array}$ & $\mathrm{P}$ & $\begin{array}{c}\text { Adjusted }^{\mathrm{a}} \mathrm{HR} \\
(95 \% \mathrm{CI})\end{array}$ & $\mathrm{P}$ & Case & $\begin{array}{c}\text { Person } \\
\text { years }\end{array}$ & $\begin{array}{c}\text { Unadjusted HR } \\
(95 \% \mathrm{CI})\end{array}$ & $\mathrm{P}$ & $\begin{array}{c}\text { Adjusted }^{\mathrm{b}} \mathrm{HR} \\
(95 \% \mathrm{CI})\end{array}$ & $\mathrm{P}$ \\
\hline & \multicolumn{6}{|c|}{ Diagnosed 2009 to 2015} & \multicolumn{6}{|c|}{ Diagnosed 2012 to 2015 (additionally adjusted for stage and grade) } \\
\hline \multicolumn{13}{|c|}{ (a) Radiotherapy after $\mathrm{ADT}^{3}$} \\
\hline Untreated & 1105 & 22590 & & & & & 511 & 10837 & 1.00 (ref. cat.) & & & \\
\hline ADT & 1608 & 21625 & $1.58(1.46,1.71)$ & $<0.001$ & $1.30(1.20,1.41)$ & $<0.001$ & 722 & 9469 & $1.67(1.49,1.88)$ & $<0.001$ & $1.31(1.10,1.57)$ & 0.002 \\
\hline ADT and radiotherapy & 752 & 15555 & $1.06(0.96,1.17)$ & 0.236 & $1.15(1.04,1.27)$ & 0.005 & 347 & 7093 & $1.11(0.96,1.29)$ & 0.143 & $1.32(1.10,1.58)$ & 0.003 \\
\hline GnRH & 1382 & 18689 & $1.57(1.45,1.71)$ & $<0.001$ & $1.31(1.21,1.42)$ & $<0.001$ & 604 & 8017 & $1.65(1.46,1.86)$ & $<0.001$ & $1.34(1.12,1.60)$ & 0.001 \\
\hline GnRH and radiotherapy & 693 & 14334 & $1.06(0.96,1.17)$ & 0.253 & $1.16(1.05,1.28)$ & 0.004 & 316 & 6558 & $1.10(0.94,1.27)$ & 0.224 & $1.31(1.09,1.58)$ & 0.004 \\
\hline Degarelix & 63 & 668 & $1.94(1.51,2.51)$ & $<0.001$ & $1.43(1.10,1.85)$ & 0.007 & 57 & 601 & $2.06(1.57,2.72)$ & $<0.001$ & $1.32(0.85,2.05)$ & 0.215 \\
\hline Degarelix and radiotherapy & 8 & 58 & $2.88(1.43,5.77)$ & 0.003 & $2.30(1.14,4.62)$ & 0.02 & 7 & 56 & $2.77(1.31,5.86)$ & 0.008 & $2.41(1.12,5.16)$ & 0.024 \\
\hline Bicalutamide & 68 & 987 & $1.47(1.15,1.88)$ & 0.002 & $1.12(0.88,1.44)$ & 0.349 & 25 & 360 & $1.52(1.02,2.27)$ & 0.041 & $1.20(0.75,1.92)$ & 0.449 \\
\hline Bicalutamide and radiotherapy & 27 & 724 & $0.82(0.56,1.21)$ & 0.315 & $0.80(0.55,1.18)$ & 0.263 & 12 & 260 & $1.05(0.59,1.87)$ & 0.864 & $1.04(0.58,1.86)$ & 0.905 \\
\hline Other antiandrogen & 99 & 1313 & $1.61(1.31,1.98)$ & $<0.001$ & $1.28(1.04,1.58)$ & 0.019 & 38 & 503 & $1.67(1.20,2.32)$ & 0.003 & $1.24(0.77,2.00)$ & 0.382 \\
\hline Other antiandrogen $+\mathrm{RT}$ & 18 & 346 & $1.13(0.71,1.81)$ & 0.602 & $1.39(0.87,2.21)$ & 0.171 & 9 & 194 & $1.05(0.54,2.03)$ & 0.885 & $1.35(0.69,2.65)$ & 0.38 \\
\hline \multicolumn{13}{|c|}{ (b) Treatment group based upon first treatment ${ }^{4}$} \\
\hline Untreated & 1105 & 22590 & 1.00 (ref. cat.) & & 1.00 (ref. cat.) & & 511 & 10837 & 1.00 (ref. cat.) & & 1.00 (ref. cat.) & \\
\hline ADT & 2323 & 36035 & $1.40(1.30,1.51)$ & $<0.001$ & $1.26(1.17,1.36)$ & $<0.001$ & 1057 & 16165 & $1.48(1.32,1.65)$ & $<0.001$ & $1.32(1.13,1.54)$ & 0.001 \\
\hline GnRH & 1836 & 29326 & $1.36(1.26,1.47)$ & $<0.001$ & $1.25(1.16,1.35)$ & $<0.001$ & 822 & 13214 & $1.41(1.25,1.58)$ & $<0.001$ & $1.30(1.11,1.53)$ & 0.001 \\
\hline Degarelix & 82 & 805 & $2.11(1.68,2.64)$ & $<0.001$ & $1.59(1.27,2.01)$ & $<0.001$ & 75 & 747 & $2.20(1.72,2.81)$ & $<0.001$ & $1.58(1.10,2.27)$ & 0.014 \\
\hline Bicalutamide & 176 & 2741 & $1.41(1.20,1.65)$ & $<0.001$ & $1.15(0.98,1.36)$ & 0.083 & 66 & 907 & $1.65(1.27,2.13)$ & $<0.001$ & $1.42(1.05,1.91)$ & 0.022 \\
\hline Other antiandrogen & 227 & 3113 & $1.59(1.37,1.83)$ & $<0.001$ & $1.31(1.13,1.51)$ & $<0.001$ & 93 & 1282 & $1.64(1.31,2.05)$ & $<0.001$ & $1.32(0.98,1.78)$ & 0.07 \\
\hline
\end{tabular}

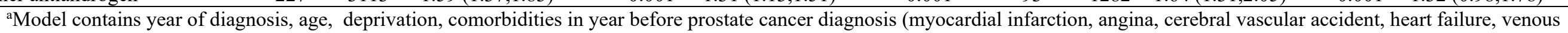

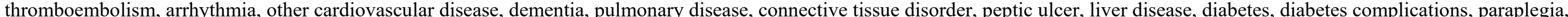

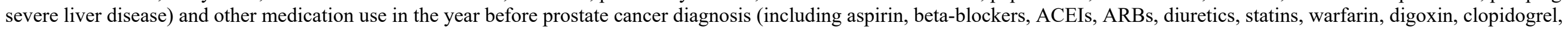

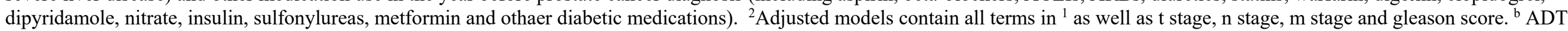

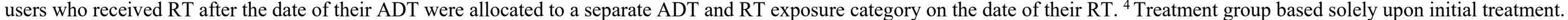




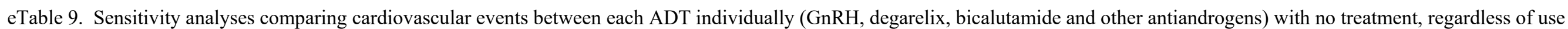
of another ADT.

\begin{tabular}{|c|c|c|c|c|c|c|c|c|c|c|c|c|c|}
\hline & & Case & $\begin{array}{c}\text { Person } \\
\text { years }\end{array}$ & $\begin{array}{c}\text { Unadjusted HR } \\
(95 \% \mathrm{CI})\end{array}$ & $\mathrm{P}$ & $\begin{array}{c}\text { Adjusted }{ }^{a} \mathrm{HR} \\
(95 \% \mathrm{CI})\end{array}$ & $\mathrm{P}$ & Case & $\begin{array}{c}\text { Person } \\
\text { years }\end{array}$ & $\begin{array}{c}\text { Unadjusted HR } \\
(95 \% \mathrm{CI})\end{array}$ & $\mathrm{P}$ & $\begin{array}{c}\text { Adjusted }{ }^{b} \mathrm{HR} \\
(95 \% \mathrm{CI})\end{array}$ & $\mathrm{P}$ \\
\hline & & \multicolumn{6}{|c|}{ Diagnosed 2009 to 2015} & \multicolumn{6}{|c|}{ Diagnosed 2012 to 2015 (additionally adjusted for stage and grade) } \\
\hline \multicolumn{2}{|l|}{ Untreated } & 1105 & 22590 & 1.00 (ref. cat.) & & 1.00 (ref. cat.) & & 511 & 10837 & 1.00 (ref. cat.) & & 1.00 (ref. cat.) & \\
\hline \multirow[t]{5}{*}{ GnRH: } & Any use & 2075 & 33023 & $1.37(1.27,1.48)$ & $<0.001$ & $1.26(1.16,1.36)$ & $<0.001$ & 920 & 14575 & $1.43(1.28,1.61)$ & $<0.001$ & $1.32(1.13,1.56)$ & 0.001 \\
\hline & 0-365 DDDs & 990 & 16606 & $1.24(1.14,1.36)$ & $<0.001$ & $1.18(1.08,1.29)$ & $<0.001$ & 487 & 8101 & $1.31(1.15,1.49)$ & $<0.001$ & $1.26(1.06,1.49)$ & 0.007 \\
\hline & $365+$ DDDs & 1085 & 16417 & $1.55(1.41,1.69)$ & $<0.001$ & $1.36(1.24,1.50)$ & $<0.001$ & 433 & 6473 & $1.65(1.43,1.90)$ & $<0.001$ & $1.55(1.26,1.89)$ & $<0.001$ \\
\hline & Current use & 1550 & 21724 & $1.52(1.40,1.64)$ & $<0.001$ & $1.33(1.23,1.44)$ & $<0.001$ & 735 & 10529 & $1.55(1.37,1.74)$ & $<0.001$ & $1.40(1.18,1.67)$ & $<0.001$ \\
\hline & Past use & 525 & 11299 & $1.03(0.92,1.15)$ & 0.61 & $1.07(0.96,1.19)$ & 0.246 & 185 & 4046 & $1.06(0.88,1.26)$ & 0.54 & $1.19(0.97,1.46)$ & 0.091 \\
\hline \multirow[t]{5}{*}{ Degaralex: } & Any use & 91 & 891 & $2.12(1.71,2.63)$ & $<0.001$ & $1.62(1.30,2.02)$ & $<0.001$ & 82 & 809 & $2.23(1.76,2.82)$ & $<0.001$ & $1.71(1.21,2.40)$ & 0.002 \\
\hline & 0-365 DDDs & 54 & 499 & $2.16(1.64,2.84)$ & $<0.001$ & $1.64(1.24,2.17)$ & $<0.001$ & 49 & 460 & $2.24(1.67,3.02)$ & $<0.001$ & $1.63(1.06,2.49)$ & 0.025 \\
\hline & 365+ DDDs & 37 & 391 & $2.07(1.49,2.88)$ & $<0.001$ & $1.60(1.15,2.23)$ & 0.006 & 33 & 349 & $2.21(1.55,3.16)$ & $<0.001$ & $1.82(1.13,2.93)$ & 0.014 \\
\hline & Current use & 71 & 744 & $1.96(1.54,2.49)$ & $<0.001$ & $1.49(1.17,1.91)$ & 0.001 & 63 & 676 & $2.03(1.56,2.64)$ & $<0.001$ & $1.42(0.95,2.12)$ & 0.086 \\
\hline & Past use & 20 & 146 & $3.00(1.93,4.68)$ & $<0.001$ & $2.38(1.52,3.71)$ & $<0.001$ & 19 & 133 & $3.35(2.11,5.31)$ & $<0.001$ & $2.80(1.64,4.78)$ & $<0.001$ \\
\hline \multirow[t]{5}{*}{ Bicalutamide: } & Any use & 299 & 4909 & $1.35(1.18,1.53)$ & $<0.001$ & $1.14(1.00,1.30)$ & 0.05 & 109 & 1542 & $1.61(1.31,1.99)$ & $<0.001$ & $1.43(1.11,1.85)$ & 0.006 \\
\hline & 0-365 DDDs & 198 & 3153 & $1.36(1.17,1.59)$ & $<0.001$ & $1.19(1.02,1.39)$ & 0.024 & 78 & 1057 & $1.66(1.30,2.11)$ & $<0.001$ & $1.58(1.18,2.11)$ & 0.002 \\
\hline & 365+ DDDs & 101 & 1756 & $1.31(1.07,1.61)$ & 0.01 & $1.05(0.85,1.29)$ & 0.666 & 31 & 485 & $1.51(1.05,2.18)$ & 0.027 & $1.17(0.77,1.78)$ & 0.471 \\
\hline & Current use & 94 & 1473 & $1.32(1.07,1.64)$ & 0.009 & $1.03(0.83,1.27)$ & 0.793 & 44 & 576 & $1.65(1.21,2.25)$ & 0.001 & $1.33(0.91,1.93)$ & 0.137 \\
\hline & Past use & 205 & 3437 & $1.36(1.16,1.58)$ & $<0.001$ & $1.20(1.03,1.40)$ & 0.018 & 65 & 966 & $1.59(1.22,2.06)$ & 0.001 & $1.50(1.10,2.04)$ & 0.01 \\
\hline \multicolumn{14}{|c|}{ Other antiandrogen: } \\
\hline & Any use & 1729 & 27419 & $1.38(1.28,1.50)$ & $<0.001$ & $1.29(1.19,1.40)$ & $<0.001$ & 783 & 12770 & $1.39(1.24,1.57)$ & $<0.001$ & $1.30(1.11,1.53)$ & 0.001 \\
\hline & 0-365 DDDs & 1628 & 26113 & $1.36(1.26,1.48)$ & $<0.001$ & $1.28(1.18,1.38)$ & $<0.001$ & 760 & 12437 & $1.39(1.23,1.56)$ & $<0.001$ & $1.30(1.10,1.53)$ & 0.002 \\
\hline & 365+ DDDs & 101 & 1307 & $1.84(1.49,2.26)$ & $<0.001$ & $1.53(1.24,1.88)$ & $<0.001$ & 23 & 333 & $1.70(1.12,2.60)$ & 0.014 & $1.84(1.14,2.99)$ & 0.013 \\
\hline & Current use & 354 & 4755 & $1.54(1.36,1.73)$ & $<0.001$ & $1.34(1.19,1.51)$ & $<0.001$ & 170 & 2268 & $1.61(1.35,1.92)$ & $<0.001$ & $1.46(1.16,1.84)$ & 0.001 \\
\hline & Past use & 1375 & 22664 & $1.34(1.23,1.46)$ & $<0.001$ & $1.28(1.17,1.39)$ & $<0.001$ & 613 & 10501 & $1.33(1.17,1.51)$ & $<0.001$ & $1.27(1.07,1.50)$ & 0.006 \\
\hline
\end{tabular}

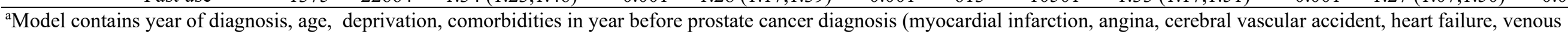

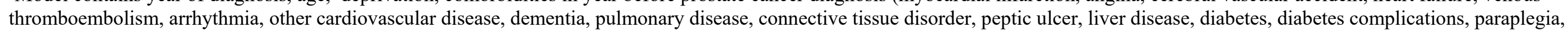

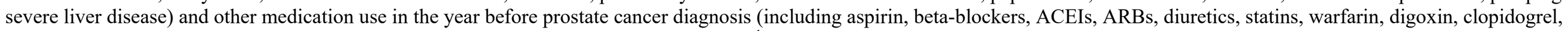

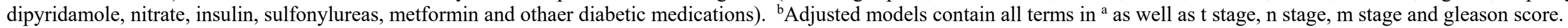




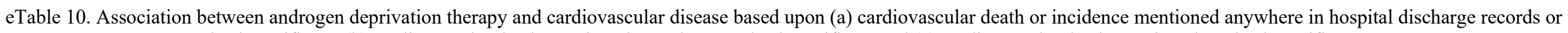
death certificate, (b) cardiovascular death mentioned anywhere on death certificate and (c) cardiovascular death mentioned on death certificate.

\begin{tabular}{|c|c|c|c|c|c|c|c|c|c|c|c|c|}
\hline & Case & $\begin{array}{c}\text { Person } \\
\text { years }\end{array}$ & $\begin{array}{c}\text { Unadjusted HR } \\
(95 \% \mathrm{CI})\end{array}$ & $\mathrm{P}$ & $\begin{array}{c}\text { Adjusted }{ }^{a} \mathrm{HR} \\
(95 \% \mathrm{CI})\end{array}$ & $\mathrm{P}$ & Case & $\begin{array}{c}\text { Person } \\
\text { years }\end{array}$ & $\begin{array}{c}\text { Unadjusted HR } \\
(95 \% \mathrm{CI})\end{array}$ & $\mathrm{P}$ & $\begin{array}{c}\text { Adjusted }^{\mathrm{b}} \mathrm{HR} \\
(95 \% \mathrm{CI})\end{array}$ & $\mathrm{P}$ \\
\hline & \multicolumn{6}{|c|}{ Diagnosed 2009 to 2015} & \multicolumn{6}{|c|}{ Diagnosed 2012 to 2015 (additionally adjusted for stage and grade) } \\
\hline & \multicolumn{12}{|c|}{ (a) Cardiovascular events (based upon cardiovascular disease anywhere in hospital discharge records and anywhere on death certificate) } \\
\hline Untreated & 2135 & 20707 & 1.00 (ref. cat.) & & 1.00 (ref. cat.) & & 1131 & 10019 & 1.00 (ref. cat.) & & 1.00 (ref. cat.) & \\
\hline ADT & 4050 & 32623 & $1.44(1.36,1.52)$ & $<0.001$ & $1.27(1.20,1.34)$ & $<0.001$ & 1922 & 14746 & $1.40(1.29,1.52)$ & $<0.001$ & $0.98(0.87,1.10)$ & 0.707 \\
\hline GnRH & 3517 & 29094 & $1.40(1.32,1.48)$ & $<0.001$ & $1.25(1.18,1.33)$ & $<0.001$ & 1628 & 13048 & $1.34(1.24,1.46)$ & $<0.001$ & $0.98(0.87,1.10)$ & 0.725 \\
\hline Degarelix & 137 & 598 & $2.34(1.97,2.78)$ & $<0.001$ & $1.64(1.37,1.95)$ & $<0.001$ & 127 & 543 & $2.30(1.91,2.77)$ & $<0.001$ & $0.97(0.73,1.29)$ & 0.814 \\
\hline Bicalutamide & 167 & 1433 & $1.34(1.14,1.57)$ & $<0.001$ & $1.07(0.91,1.26)$ & 0.396 & 71 & 530 & $1.43(1.12,1.82)$ & 0.004 & $0.95(0.72,1.25)$ & 0.719 \\
\hline Other antiandrogen & 224 & 1447 & $1.76(1.53,2.02)$ & $<0.001$ & $1.48(1.29,1.70)$ & $<0.001$ & 95 & 614 & $1.65(1.33,2.03)$ & $<0.001$ & $1.03(0.76,1.38)$ & 0.863 \\
\hline \multicolumn{13}{|c|}{ (b) Cardiovascular death (based upon cardiovascular disease anywhere on death certificate) } \\
\hline Untreated & 551 & 24580 & 1.00 (ref. cat.) & & 1.00 (ref. cat.) & & 257 & 11509 & 1.00 (ref. cat.) & & 1.00 (ref. cat.) & \\
\hline ADT & 1635 & 41954 & $1.92(1.73,2.12)$ & $<0.001$ & $1.64(1.48,1.81)$ & $<0.001$ & 717 & 18175 & $2.00(1.71,2.33)$ & $<0.001$ & $1.23(0.91,1.67)$ & 0.183 \\
\hline GnRH & 1406 & 37285 & $1.85(1.66,2.05)$ & $<0.001$ & $1.62(1.46,1.80)$ & $<0.001$ & 586 & 15971 & $1.84(1.57,2.16)$ & $<0.001$ & $1.26(0.92,1.71)$ & 0.148 \\
\hline Degarelix & 85 & 832 & $4.90(3.89,6.18)$ & $<0.001$ & $2.84(2.23,3.61)$ & $<0.001$ & 78 & 753 & $5.13(3.96,6.64)$ & $<0.001$ & $1.40(0.82,2.41)$ & 0.22 \\
\hline Bicalutamide & 58 & 1908 & $1.48(1.13,1.94)$ & 0.005 & $1.20(0.91,1.58)$ & 0.186 & 18 & 676 & $1.34(0.83,2.16)$ & 0.239 & $0.89(0.44,1.79)$ & 0.744 \\
\hline Other antiandrogen & 82 & 1864 & $2.13(1.69,2.70)$ & $<0.001$ & $1.59(1.26,2.02)$ & $<0.001$ & 34 & 763 & $2.23(1.55,3.20)$ & $<0.001$ & $1.06(0.55,2.04)$ & 0.858 \\
\hline \multicolumn{13}{|c|}{ (c) Cardiovascular incidence (based upon cardiovascular disease anywhere in hospital discharge records) } \\
\hline Untreated & 1964 & 20707 & 1.00 (ref. cat.) & & 1.00 (ref. cat.) & & 1044 & 10019 & 1.00 (ref. cat.) & & 1.00 (ref. cat.) & \\
\hline ADT & 3606 & 32623 & $1.39(1.31,1.47)$ & $<0.001$ & $1.23(1.16,1.31)$ & $<0.001$ & 1704 & 14746 & $1.34(1.23,1.46)$ & $<0.001$ & $0.97(0.87,1.10)$ & 0.668 \\
\hline GnRH & 3140 & 29094 & $1.36(1.28,1.44)$ & $<0.001$ & $1.22(1.15,1.30)$ & $<0.001$ & 1455 & 13048 & $1.29(1.19,1.41)$ & $<0.001$ & $0.98(0.87,1.10)$ & 0.722 \\
\hline Degarelix & 105 & 598 & $1.94(1.60,2.37)$ & $<0.001$ & $1.39(1.13,1.69)$ & 0.001 & 97 & 543 & $1.89(1.53,2.33)$ & $<0.001$ & $0.88(0.65,1.21)$ & 0.434 \\
\hline Bicalutamide & 153 & 1433 & $1.33(1.13,1.57)$ & 0.001 & $1.07(0.90,1.26)$ & 0.445 & 65 & 530 & $1.41(1.09,1.81)$ & 0.008 & $0.93(0.70,1.25)$ & 0.646 \\
\hline Other antiandrogen & 204 & 1447 & $1.74(1.50,2.01)$ & $<0.001$ & $1.48(1.28,1.72)$ & $<0.001$ & 86 & 614 & $1.61(1.29,2.01)$ & $<0.001$ & $1.05(0.78,1.43)$ & 0.739 \\
\hline
\end{tabular}

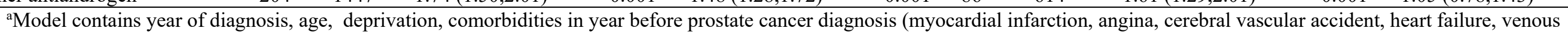

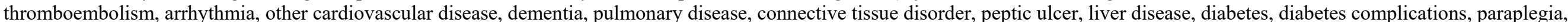

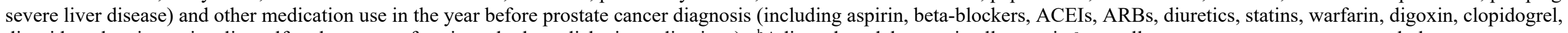

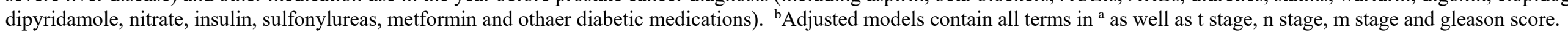


eTable 11. Association between current use of androgen deprivation therapy and cardiovascular events by type in patients diagnosed 2009 to 2015.

\begin{tabular}{|c|c|c|c|c|c|c|c|}
\hline & & Case & $\begin{array}{c}\text { Person } \\
\text { years }\end{array}$ & $\begin{array}{c}\text { Unadjusted HR } \\
(95 \% \mathrm{CI})\end{array}$ & $\mathrm{P}$ & Adjusted $^{\mathrm{a}} \mathrm{HR}(95 \% \mathrm{CI})$ & $\mathrm{P}$ \\
\hline \multicolumn{8}{|c|}{ Angina } \\
\hline \multicolumn{2}{|l|}{ Untreated } & 260 & 24089 & 1.00 (ref. cat.) & & 1.00 (ref. cat.) & \\
\hline \multirow[t]{2}{*}{ ADT: } & Current use & 353 & 26434 & $1.19(1.01,1.40)$ & 0.042 & $1.02(0.87,1.21)$ & 0.774 \\
\hline & Past use & 157 & 14489 & $1.01(0.82,1.24)$ & 0.933 & $1.02(0.83,1.26)$ & 0.844 \\
\hline \multirow[t]{2}{*}{ GnRH: } & Current use & 301 & 23705 & $1.13(0.95,1.33)$ & 0.174 & $1.00(0.84,1.18)$ & 0.977 \\
\hline & Past use & 139 & 12665 & $1.02(0.82,1.27)$ & 0.832 & $1.04(0.84,1.30)$ & 0.695 \\
\hline \multirow[t]{2}{*}{ Degaralex: } & Current use & 14 & 768 & $1.61(0.94,2.75)$ & 0.086 & $1.02(0.59,1.76)$ & 0.945 \\
\hline & Past use & $<5$ & 51 & $3.40(0.85,13.70)$ & 0.085 & $1.60(0.40,6.49)$ & 0.507 \\
\hline \multicolumn{2}{|c|}{ Bicalutamide: Current use } & 20 & 1045 & $1.71(1.08,2.70)$ & 0.021 & $1.23(0.78,1.95)$ & 0.369 \\
\hline & Past use & 10 & 809 & $1.16(0.61,2.19)$ & 0.647 & $0.96(0.51,1.82)$ & 0.91 \\
\hline \multicolumn{8}{|c|}{ Other antiandrogen: } \\
\hline & Current use & 11 & 547 & $1.85(1.01,3.38)$ & 0.046 & $1.44(0.79,2.65)$ & 0.236 \\
\hline & Past use & 13 & 1268 & $0.92(0.53,1.61)$ & 0.776 & $0.96(0.55,1.68)$ & 0.893 \\
\hline \multicolumn{8}{|c|}{ Myocardial infarction } \\
\hline \multicolumn{2}{|l|}{ Untreated } & 201 & 24308 & 1.00 (ref. cat.) & & 1.00 (ref. cat.) & \\
\hline \multirow[t]{2}{*}{ ADT: } & Current use & 336 & 26610 & $1.62(1.35,1.94)$ & $<0.001$ & $1.35(1.12,1.62)$ & 0.001 \\
\hline & Past use & 104 & 14742 & $0.88(0.69,1.14)$ & 0.336 & $0.92(0.72,1.19)$ & 0.534 \\
\hline \multirow[t]{2}{*}{ GnRH: } & Current use & 300 & 23857 & $1.62(1.34,1.94)$ & $<0.001$ & $1.37(1.13,1.65)$ & 0.001 \\
\hline & Past use & 86 & 12902 & $0.83(0.64,1.09)$ & 0.18 & $0.88(0.68,1.15)$ & 0.361 \\
\hline \multirow[t]{2}{*}{ Degaralex: } & Current use & 12 & 769 & $2.01(1.12,3.60)$ & 0.02 & $1.38(0.76,2.50)$ & 0.29 \\
\hline & Past use & $<5$ & 50 & & & & \\
\hline \multicolumn{2}{|c|}{ Bicalutamide: Current use } & 14 & 1048 & $1.71(0.99,2.94)$ & 0.054 & $1.26(0.73,2.17)$ & 0.412 \\
\hline & Past use & 12 & 820 & $1.81(1.01,3.27)$ & 0.048 & $1.76(0.97,3.17)$ & 0.061 \\
\hline \multicolumn{8}{|c|}{ Other antiandrogen: } \\
\hline & Current use & 6 & 559 & $1.34(0.59,3.02)$ & 0.483 & $0.94(0.42,2.13)$ & 0.884 \\
\hline & Past use & 10 & 1282 & $1.00(0.53,1.90)$ & 0.992 & $0.95(0.50,1.80)$ & 0.87 \\
\hline \multicolumn{8}{|c|}{ Arrhythmia } \\
\hline Untreated & & 184 & 24221 & 1.00 (ref. cat.) & & 1.00 (ref. cat.) & \\
\hline ADT: & Current use & 301 & 26447 & $1.51(1.25,1.82)$ & $<0.001$ & $1.30(1.07,1.57)$ & 0.007 \\
\hline & Past use & 113 & 14617 & $0.98(0.77,1.25)$ & 0.87 & $1.02(0.79,1.30)$ & 0.899 \\
\hline GnRH: & Current use & 262 & 23729 & $1.46(1.20,1.77)$ & $<0.001$ & $1.28(1.05,1.56)$ & 0.013 \\
\hline & Past use & 95 & 12772 & $0.94(0.72,1.21)$ & 0.625 & $0.98(0.75,1.27)$ & 0.874 \\
\hline Degaralex: & Current use & 12 & 756 & $2.14(1.19,3.84)$ & 0.011 & $1.46(0.80,2.66)$ & 0.215 \\
\hline & Past use & $<5$ & 53 & $4.90(1.21,19.76)$ & 0.026 & $3.39(0.83,13.76)$ & 0.088 \\
\hline Bicalutamide & e: Current use & 11 & 1043 & $1.41(0.77,2.59)$ & 0.271 & $1.11(0.60,2.05)$ & 0.737 \\
\hline & Past use & 6 & 837 & $0.89(0.39,2.02)$ & 0.788 & $0.86(0.38,1.96)$ & 0.723 \\
\hline Other antian & drogen: & & & & & & \\
\hline & Current use & 9 & 542 & $2.23(1.14,4.35)$ & 0.019 & $1.80(0.92,3.53)$ & 0.087 \\
\hline & Past use & 16 & 1268 & $1.64(0.98,2.74)$ & 0.06 & $1.55(0.93,2.60)$ & 0.094 \\
\hline & & & & Heart failure & & & \\
\hline Untreated & & 104 & 24454 & 1.00 (ref. cat.) & & 1.00 (ref. cat.) & \\
\hline ADT: & Current use & 193 & 26699 & $1.77(1.38,2.27)$ & $<0.001$ & $1.35(1.05,1.73)$ & 0.02 \\
\hline & Past use & 36 & 14939 & $0.62(0.42,0.93)$ & 0.02 & $0.78(0.52,1.16)$ & 0.221 \\
\hline GnRH: & Current use & 172 & 23944 & $1.77(1.37,2.28)$ & $<0.001$ & $1.38(1.07,1.78)$ & 0.014 \\
\hline & Past use & 25 & 13074 & $0.49(0.31,0.78)$ & 0.002 & $0.64(0.41,1.01)$ & 0.058 \\
\hline Degaralex: & Current use & 7 & 766 & $2.19(1.01,4.72)$ & 0.046 & $1.20(0.54,2.65)$ & 0.654 \\
\hline & Past use & $<5$ & 54 & $4.82(0.67,34.62)$ & 0.118 & $2.20(0.30,16.04)$ & 0.435 \\
\hline Bicalutamide & e: Current use & 10 & 1049 & $2.33(1.21,4.46)$ & 0.011 & $1.70(0.88,3.28)$ & 0.114 \\
\hline & Past use & 6 & 832 & $1.86(0.81,4.27)$ & 0.145 & $2.23(0.97,5.16)$ & 0.06 \\
\hline Other antian & drogen: & & & & & & \\
\hline & Current use & $<5$ & 560 & $0.43(0.06,3.07)$ & 0.399 & $0.24(0.03,1.71)$ & 0.153 \\
\hline & Past use & 7 & 1294 & $1.38(0.64,2.98)$ & 0.411 & $1.21(0.56,2.62)$ & 0.634 \\
\hline & & & & Stroke & & & \\
\hline Untreated & & 226 & 24198 & 1.00 (ref. cat.) & & 1.00 (ref. cat.) & \\
\hline ADT: & Current use & 312 & 26491 & $1.29(1.08,1.54)$ & 0.005 & $1.07(0.90,1.28)$ & 0.45 \\
\hline & Past use & 125 & 14715 & $0.89(0.71,1.12)$ & 0.335 & $0.95(0.76,1.20)$ & 0.683 \\
\hline GnRH: & Current use & 286 & 23754 & $1.32(1.10,1.58)$ & 0.003 & $1.11(0.92,1.33)$ & 0.272 \\
\hline & Past use & 108 & 12866 & $0.88(0.69,1.12)$ & 0.303 & $0.95(0.75,1.22)$ & 0.705 \\
\hline Degaralex: & Current use & 6 & 764 & $0.88(0.39,1.97)$ & 0.748 & $0.62(0.28,1.42)$ & 0.26 \\
\hline & Past use & $<5$ & 54 & & & & \\
\hline
\end{tabular}




\begin{tabular}{|c|c|c|c|c|c|c|c|}
\hline \multicolumn{2}{|c|}{ Bicalutamide: Current use } & 8 & 1046 & $0.84(0.42,1.71)$ & 0.632 & $0.68(0.34,1.38)$ & 0.285 \\
\hline & Past use & $<5$ & 838 & $0.49(0.18,1.33)$ & 0.164 & $0.53(0.20,1.44)$ & 0.213 \\
\hline \multicolumn{8}{|c|}{ Other antiandrogen: } \\
\hline & Current use & 7 & 549 & $1.39(0.65,2.95)$ & 0.393 & $1.00(0.47,2.14)$ & 0.994 \\
\hline & Past use & 17 & 1272 & $1.44(0.88,2.36)$ & 0.151 & $1.27(0.77,2.10)$ & 0.339 \\
\hline \multicolumn{8}{|c|}{ Venous thromboembolism } \\
\hline \multirow{3}{*}{$\begin{array}{l}\text { Untreated } \\
\text { ADT: }\end{array}$} & & 89 & 24459 & 1.00 (ref. cat.) & & 1.00 (ref. cat.) & \\
\hline & Current use & 234 & 26596 & $2.95(2.28,3.82)$ & $<0.001$ & $2.89(2.22,3.75)$ & $<0.001$ \\
\hline & Past use & 39 & 14864 & $0.89(0.60,1.32)$ & 0.561 & $0.91(0.61,1.36)$ & 0.645 \\
\hline \multirow[t]{2}{*}{ GnRH: } & Current use & 210 & 23844 & $2.98(2.29,3.88)$ & $<0.001$ & $2.93(2.24,3.82)$ & $<0.001$ \\
\hline & Past use & 33 & 12993 & $0.86(0.56,1.31)$ & 0.482 & $0.89(0.58,1.35)$ & 0.58 \\
\hline \multirow[t]{2}{*}{ Degaralex: } & Current use & 9 & 760 & $3.84(1.92,7.67)$ & $<0.001$ & $3.73(1.85,7.53)$ & $<0.001$ \\
\hline & Past use & $<5$ & 54 & $6.87(0.95,49.53)$ & 0.056 & $7.63(1.05,55.37)$ & 0.045 \\
\hline \multicolumn{2}{|c|}{ Bicalutamide: Current use } & $<5$ & 1056 & $0.92(0.29,2.91)$ & 0.886 & $0.83(0.26,2.65)$ & 0.758 \\
\hline & Past use & $<5$ & 846 & $0.80(0.20,3.29)$ & 0.762 & $0.81(0.20,3.32)$ & 0.771 \\
\hline \multicolumn{8}{|c|}{ Other antiandrogen: } \\
\hline & Current use & 6 & 558 & $3.10(1.35,7.10)$ & 0.007 & $2.98(1.29,6.85)$ & 0.01 \\
\hline & Past use & 9 & 1285 & $2.44(1.22,4.89)$ & 0.012 & $2.36(1.17,4.74)$ & 0.016 \\
\hline \multicolumn{8}{|c|}{ Other CVD events } \\
\hline \multicolumn{2}{|l|}{ Untreated } & 320 & 23967 & 1.00 (ref. cat.) & & 1.00 (ref. cat.) & \\
\hline \multirow[t]{2}{*}{ ADT: } & Current use & 484 & 26157 & $1.41(1.22,1.63)$ & $<0.001$ & $1.24(1.07,1.44)$ & 0.004 \\
\hline & Past use & 242 & 14233 & $1.32(1.10,1.57)$ & 0.002 & $1.36(1.14,1.63)$ & 0.001 \\
\hline \multirow[t]{2}{*}{ GnRH: } & Current use & 429 & 23449 & $1.39(1.20,1.62)$ & $<0.001$ & $1.24(1.07,1.44)$ & 0.005 \\
\hline & Past use & 228 & 12403 & $1.43(1.19,1.71)$ & $<0.001$ & $1.50(1.25,1.80)$ & $<0.001$ \\
\hline \multirow[t]{2}{*}{ Degaralex: } & Current use & 22 & 754 & $2.21(1.43,3.41)$ & $<0.001$ & $1.82(1.17,2.83)$ & 0.008 \\
\hline & Past use & $<5$ & 50 & & & & \\
\hline \multicolumn{2}{|c|}{ Bicalutamide: Current use } & 9 & 1033 & $0.66(0.34,1.29)$ & 0.223 & $0.54(0.28,1.05)$ & 0.07 \\
\hline & Past use & 10 & 810 & $0.97(0.51,1.82)$ & 0.917 & $0.96(0.51,1.82)$ & 0.912 \\
\hline \multicolumn{8}{|c|}{ Other antiandrogen: } \\
\hline & Current use & 15 & 555 & $2.07(1.23,3.48)$ & 0.006 & $1.56(0.92,2.62)$ & 0.097 \\
\hline & Past use & 13 & 1271 & $0.78(0.45,1.36)$ & 0.385 & $0.73(0.42,1.27)$ & 0.266 \\
\hline
\end{tabular}

${ }^{a}$ Model contains for year of diagnosis, age, deprivation, comorbidities in year before prostate cancer diagnosis (myocardial infarction, angina, cerebral vascular accident, heart failure, venous thromboembolism, arrhythmia, other cardiovascular disease, dementia, pulmonary disease, connective tissue disorder, peptic ulcer, liver disease, diabetes, diabetes complications, paraplegia, severe liver disease) and other medication use in the year before prostate cancer diagnosis (including aspirin, beta-blockers, ACEIs, ARBs, diuretics, statins, warfarin, digoxin, clopidogrel, dipyridamole, nitrate, insulin, sulfonylureas, metformin and othaer diabetic medications). 